\title{
Time and Outcome Framing in Intertemporal Tradeoffs
}

\author{
Marc Scholten \\ ISPA University Institute
}

\author{
Daniel Read \\ University of Warwick
}

\begin{abstract}
A robust anomaly in intertemporal choice is the delay-speedup asymmetry: Receipts are discounted more, and payments are discounted less, when delayed than when expedited over the same interval. We developed 2 versions of the tradeoff model (Scholten \& Read, 2010) to address such situations, in which an outcome is expected at a given time but then its timing is changed. The outcome framing model generalizes the approach taken by the hyperbolic discounting model (Loewenstein \& Prelec, 1992): Not obtaining a positive outcome when expected is a worse than expected state, to which people are over-responsive, or hypersensitive, and not incurring a negative outcome when expected is a better than expected state, to which people are under-responsive, or hyposensitive. The time framing model takes a new approach: Delaying a positive outcome or speeding up a negative one involves a loss of time to which people are hypersensitive, and speeding up a positive outcome or delaying a negative one involves a gain of time to which people are hyposensitive. We compare the models on their quantitative predictions of indifference data from matching and preference data from choice. The time framing model systematically outperforms the outcome framing model.
\end{abstract}

Keywords: intertemporal choice, discounting, delay-speedup asymmetry, outcome framing, time framing

Intertemporal choices are choices between streams of outcomes differing in value and timing. Psychologists have largely investigated monetary intertemporal choices, such as one between $\$ 100$ now and $\$ 150$ in 1 year. This is a decision of whether to accept or decline a $50 \%$ interest rate applied to $\$ 100$ now, or, conversely, of whether to accept or decline a $33 \%$ discount rate applied to $\$ 150$ in 1 year. Psychologists have discovered numerous anomalies to a rationalchoice analysis, in which the interest or discount rate implied by choice is constant and equal to the decision maker's opportunity cost of money. For many people, this is the bank rate of interest or the mortgage rate. However, people are influenced by many characteristics of the choice task that are normatively irrelevant.

Every monetary transaction will involve receipts or payments. To determine the interest rate, rational-choice analysis does not care which. Yet, experimental investigations have revealed two robust patterns in which receipts and payments are not treated in the same way. One result, the gain-loss asymmetry (Loewenstein \& Prelec, 1992) or the sign effect (Thaler, 1981), is a main effect of outcome sign: People offer lower interest rates for payments than they demand for receipts (Baker, Johnson, \& Bickel, 2003; McAlvanah, 2010;

This article was published Online First January 28, 2013.

Marc Scholten, Department of Social and Organizational Psychology, ISPA University Institute, Lisbon, Portugal; Daniel Read, Warwick Business School, University of Warwick, Coventry, England.

We acknowledge the financial support from the Fundação para a Ciência e Tecnologia (FCT), Program POCI 2010, and Projects POCI/ PSI/56030/2004, PPCDT/PSI/56030/2004, and PTDC/PSI-PCO/101447/ 2008.

Correspondence concerning this article should be addressed to Marc Scholten, ISPA University Institute, Rua Jardim do Tabaco 34, 1149-041 Lisboa, Portugal. E-mail: scholten@ispa.pt
Murphy, Vuchinich, \& Simpson, 2001; Xu, Liang, Wang, Li, \& Jiang, 2009; Yates \& Watts, 1975). Consider the following two choices between a smaller-sooner outcome (SS) and a larger-later one $(L L)$ :

\section{Receipts: \\ SS Receive $\$ 100$ today. \\ LL Receive $\$ 150$ in 1 year.}

Payments:

SS Pay $\$ 100$ today.

$L L \quad$ Pay $\$ 150$ in 1 year.

Someone who is indifferent between $S S$ and $L L$ for receipts (demanding a $50 \%$ interest rate) will typically prefer $S S$ for payments (offering less than 50\%). The other result, the delayspeedup asymmetry (Loewenstein \& Prelec, 1992), is an interaction effect between outcome sign and changes in outcome timing: People demand higher interest rates for delayed receipts than they offer for expedited ones (Appelt, Hardisty, \& Weber, 2011; Benzion, Rapoport, \& Yagil, 1989; Loewenstein, 1988; Malkoc \& Zauberman, 2006; McAlvanah, 2010; Shelley, 1993; Weber et al., 2007) and offer lower interest rates for delayed payments than they demand for expedited ones (Appelt et al., 2011; Benzion et al., 1989; McAlvanah, 2010; Shelley, 1993). Consider the following pairs of choices for receipts and payments:

\section{Receipts:}

Delay:

You are entitled to receive $\$ 100$ today. Choose between:

SS Receive \$100 today, as planned.

$L L$ Delay the receipt and receive $\$ 200$ in 1 year instead. 
Speedup:

You are entitled to receive $\$ 200$ in 1 year. Choose between:

SS Receive \$200 in 1 year, as planned.

$L L \quad$ Speed up the receipt and receive $\$ 100$ today instead.

Payments:

Delay:

You are obliged to pay $\$ 100$ today. Choose between:

SS Pay $\$ 100$ today, as planned.

LL Delay the payment and pay $\$ 120$ in 1 year instead.

Speedup:

You are obliged to pay $\$ 120$ in 1 year. Choose between:

SS Pay $\$ 120$ in 1 year, as planned.

$L L \quad$ Speed up the payment and pay $\$ 100$ today instead.

Someone who, for receipts, is indifferent between $S S$ and $L L$ in the delay frame (demanding a $100 \%$ interest rate) will typically prefer $L L$ in the speedup frame (offering less than 100\%). In a similar vein, someone who, for payments, is indifferent between $S S$ and $L L$ in the delay frame (offering a $20 \%$ interest rate) will typically prefer $L L$ in the speedup frame (demanding more than 20\%). "Because," according to Loewenstein and Prelec (1992, p. 578), "the two pairs of choices are actually different representations of the same underlying options, the [preference patterns] constitute a classic framing effect, which is inconsistent with any normative theory." In this article, we examine two alternative interpretations of the delay-speedup asymmetry, one ascribing it to the framing of outcomes, as is done in previous models (Loewenstein, 1988; Loewenstein \& Prelec, 1992; Shelley, 1993), and the other ascribing it to the framing of time.

The gain-loss and delay-speedup asymmetries, however, are not the only anomalies to the normative interest-rate model. To accommodate a broad range of anomalies, many of which are also anomalous to "conventional" models like the discounted utility model (P. A. Samuelson, 1937), the hyperbolic discounting model (Loewenstein \& Prelec, 1992), and the quasihyperbolic discounting model (Laibson, 1997), we developed the tradeoff model (Scholten \& Read, 2010). In this article, we further develop the tradeoff model to arrive at quantitative predictions of data that contain the gain-loss and delayspeedup asymmetries along with three other anomalies. In particular, we develop two versions of the tradeoff model, one that ascribes the delay-speedup asymmetry to outcome framing, and the other that ascribes it to time framing. The time framing model systematically outperforms the outcome framing model in the prediction of indifference data from matching and preference data from choice.

We begin with the compound-interest model and the exponential discounting model deriving from it, which will provide the dependent variables in our analyses of indifference data from matching. The exponential discounting model, along with other discounting models to be specified later in this introduction, will serve as benchmarks to evaluate the predictive accuracy of the tradeoff models.

\section{Compound Interest Rates and Exponential Discount Rates}

Let $x_{S}$ be the immediate outcome, and $x_{L}$ be the outcome that is delayed by $t_{L}$ units of time, so that the smaller-sooner ( $S S$ ) outcome is $\left(x_{S}, 0\right)$ and the larger-later $(L L)$ outcome is $\left(x_{L}, t_{L}\right)$. The interest-rate model posits that the person will be indifferent between $S S$ and $L L$ when $x_{L}$ is the amount that $x_{S}$ would become if increased by the interest over the period $t_{L}$. Thus, when the personal interest rate is $r$, we have:

$$
x_{S}(1+r)^{t_{L}}=x_{L}
$$

In this model, the person demands compound interest, which is that interest earned over $x_{S}$ also bears interest. To illustrate, someone indifferent between $\$ 100$ today and $\$ 150$ in 1 year will also be indifferent between $\$ 100$ and $\$ 225$ in 2 years. Solving Equation 1 for the per-period interest rate,

$$
r=\left(\frac{x_{L}}{x_{S}}\right)^{1 / t_{L}}-1
$$

The compound-interest model describes present-to-future conversion, but it may be changed so as to describe future-topresent conversion instead, which is what the exponential discounting model does:

$$
x_{S}=\frac{1}{(1+r)^{t_{L}}} x_{L}=\delta^{t_{L}} x_{L}
$$

where $0<\delta<1$ is a per-period discount factor, which indicates the proportion of the (discounted) value of $x_{L}$ that remains over one period of time. To illustrate, if the person is indifferent between $\$ 100$ today and $\$ 150$ in 1 year, the proportion of the $\$ 150$ receipt that remains over a period of 1 year is $67 \%$. Solving Equation 3 for the per-period discount factor,

$$
\delta=\left(\frac{x_{S}}{x_{L}}\right)^{1 / t_{L}} .
$$

The per-period discount factor is inversely related to discounting: Lower values indicate more discounting. Instead of looking at how much remains over one period of time, one can look at how much is lost over one period of time. This is what the per-period discount rate does: It indicates the proportion of the value of $x_{L}$ that is lost over one period of time:

$$
\rho=\frac{r}{1+r}=1-\delta
$$

where $0<\rho<1$ is the per-period discount rate. To illustrate, if the person is indifferent between $\$ 100$ today and $\$ 150$ in 1 year, the rate at which the $\$ 150$ receipt is discounted is $\rho=$ $.50 /(1+.50)=1-.67=.33$, that is, the proportion of its value that is lost over one period of time is $33 \%$. The per-period discount rate is directly related to discounting, in that higher values indicate more discounting.

The exponential discounting model posits that the personal interest rate $r$, or the discount fraction $\delta$ and discount rate $\rho$ into which the personal interest rate can be transformed, is constant. However, the gain-loss and delay-speedup asymmetries show that $r$ varies. In the next section, we describe three other anomalies to the exponential discounting model that will feature in our data. 


\section{Anomalies to the Exponential Discounting Model}

Two anomalies were described in the introduction:

1. The gain-loss asymmetry or the sign effect, and

2. the delay-speedup asymmetry.

In addition to these sign-related anomalies, we examine anomalies related to the magnitude of the outcomes and the delay to the outcomes, which are described below.

3. The absolute magnitude effect (Loewenstein \& Prelec, 1992) or the size effect (see Thaler, 1981): People demand or offer lower interest rates with large outcomes than with small ones. For instance, someone indifferent between $\$ 100$ today and $\$ 150$ in 1 year (50\%) might also be indifferent between $\$ 1,000$ today and $\$ 1,250$ in 1 year $(25 \%)$. The absolute magnitude effect is probably the most robust anomaly in intertemporal choice (Scholten \& Read, 2010), and the most difficult one to address with parametric specifications of discounting models (Scholten \& Read, 2012).

4. The delay effect (Thaler, 1981): People demand or offer lower interest rates with long delays than with short ones. For instance, someone indifferent between $\$ 100$ today and $\$ 150$ in 1 year (50\% per annum) might also be indifferent between $\$ 100$ today and $\$ 200$ in 2 years (41\% per annum). The delay effect is conventionally addressed by hyperbolic discounting models.

Finally, while the delay-speedup asymmetry is an interaction between outcome sign and changes in outcome timing, we also examine its interaction with delay length. This anomaly has not been identified earlier, but we infer it from one piece of available evidence.

5. An attenuation of the delay-speedup asymmetry for longer delays. The evidence comes from Malkoc and Zauberman (2006), who reported an interaction effect between the delay to the larger outcome and changes in outcome timing: The delay effect was stronger when delaying a receipt than when speeding it up. A hypothetical reconstruction of their result is provided in the top left panel of Figure 1. The dependent variable is the interest rate $r$, which, for comparability with subsequent presentations of results, is reversely scaled. We see that $r$ is higher in the delay frame than in the speedup frame, which is the delay-speedup asymmetry for receipts, and that $r$ decreases more sharply with delay length in the delay frame than in the speedup frame, which is the novel result: The strength of the delay effect depends on the frame.

Malkoc and Zauberman (2006) did not examine how their result would generalize to payments, but our prediction is that it will actually reverse for payments: We expect that $r$ will be higher in the speedup frame than in the delay frame, which is the delay-speedup asymmetry for payments, and that $r$ will decrease more sharply with delay length in the speedup frame than in the delay frame, a hypothetical representation of which is given in the top right panel of Figure 1.

To see the rationale for our prediction, the bottom panels of Figure 1 represent the same results in a different format, with the respective panels representing short and long delays rather than receipts and payments. What we see is an attenuation of the delay-speedup asymmetry for longer delays. That is, $r$ generally decreases for longer delays, which is the delay effect, but also becomes less affected by changes in outcome timing, which, according to the tradeoff model, is a corollary of the delay effect.

\section{The Basic Tradeoff Model}

Consider a sooner outcome $x_{S}$ delayed by $t_{S}$ units of time, and a larger outcome $x_{L}$ delayed by $t_{L}$ units of time. In the present investigation, we consider only cases where the sooner outcome is immediate, so that $t_{S}=0$. When the outcomes are positive (e.g., receiving $\$ 100$ today or $\$ 150$ in 1 year), $S S$ has an advantage along the time attribute (one will receive 1 year sooner), while $L L$ has an advantage along the outcome attribute (one will receive $\$ 50$ more). When the outcomes are negative (e.g., paying $\$ 100$ today or $\$ 150$ in 1 year), $S S$ has an advantage along the outcome attribute (one will pay $\$ 50$ less), while $L L$ has an advantage along the time attribute (one will pay 1 year later).

Advantages do not enter the model as differences between raw attribute amounts, but rather as differences between amounts that are transformed to take the psychological impact of time and outcome into account. Specifically, advantages are differences between weighted delays, $w\left(t_{L}\right)-w(0)=w\left(t_{L}\right)$, and between valued outcomes, $v\left(x_{L}\right)-v\left(x_{S}\right)$ for receipts and $v\left(x_{S}\right)-v\left(x_{L}\right)$ for payments. The decision maker will prefer $S S$ when its advantage is greater than that of $L L$, prefer $L L$ when its advantage is greater than that of $S S$, and be indifferent otherwise. The indifference point is given as follows:

$$
\kappa w\left(t_{L}\right)= \begin{cases}v\left(x_{L}\right)-v\left(x_{S}\right) & \text { if } x_{L}>x_{S}>0 \\ v\left(x_{S}\right)-v\left(x_{L}\right) & \text { if } x_{L}<x_{S}<0,\end{cases}
$$

where $\kappa>0$ is a tradeoff parameter, which scales the difference between weighted delays and the difference between valued outcomes in a common currency.

The value function $v$ and the time-weighing function $w$ are reference-dependent functions ranging from identity functions, that is, $v(x)=x$ and $w(t)=t$ (constant sensitivity), to zero functions, that is, $v(x)=0$ for all $x$, and $w(t)=0$ for all $t$ (insensitivity). Between these two limits, $v$ and $w$ are concave functions, thus exhibiting diminishing absolute sensitivity (Tversky \& Kahneman, 1991): For constant absolute increases of $x$ and $t, v(x)$ and $w(t)$ increase by decreasing absolute amounts. For instance, adding 1 day to a delay of 1 week yields a greater increase in the weight of time than adding 1 day to a delay of 52 weeks. Furthermore, $v$ and $w$ exhibit augmenting proportional sensitivity (Scholten \& Read, 2010): For constant proportional increases of $x$ and $t, w(t)$ and $v(x)$ increase by increasing absolute amounts. For instance, doubling $\$ 100$ yields a greater increase in value than doubling $\$ 1$. Finally, $v$ exhibits constant loss aversion (Tversky \& Kahneman, 1991): Reversing the sign of an outcome from positive to negative increases the magnitude of its value by a multiplicative constant, that is, $v(-x)=-\lambda v(x)$, where $\lambda>1$ and $x>0$.

The tradeoff model in Equation 5 accommodates all of the anomalies described above, except for the delay-speedup asymmetry, which is why we refer to it as the basic tradeoff model. In 

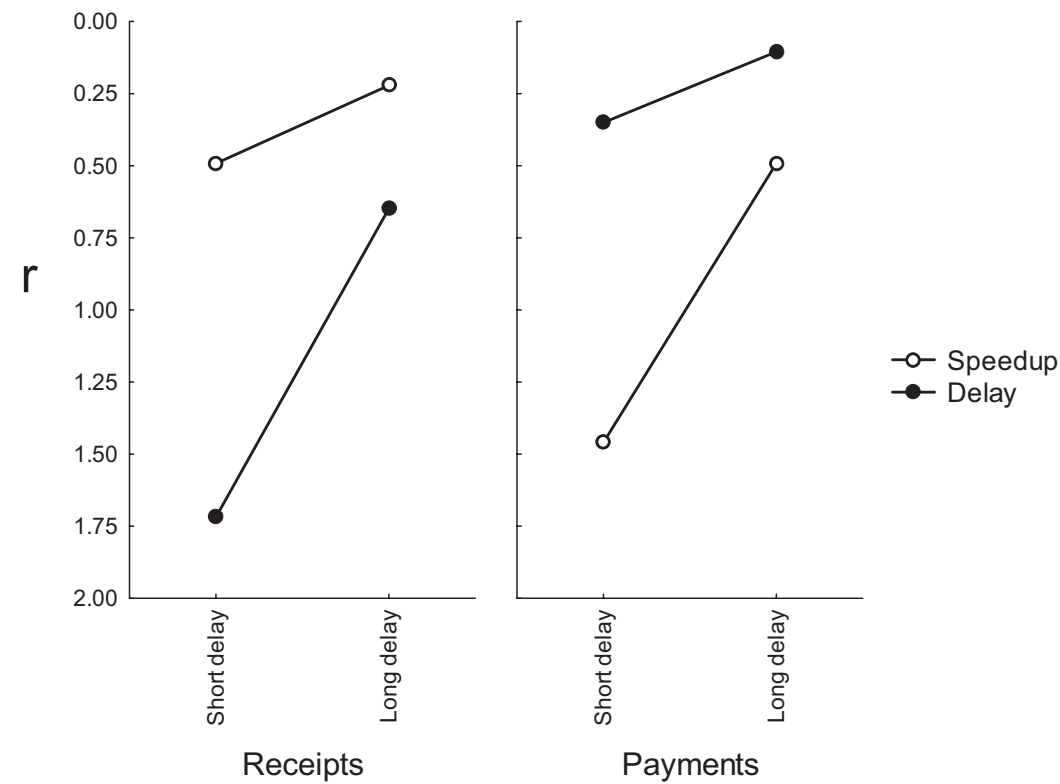

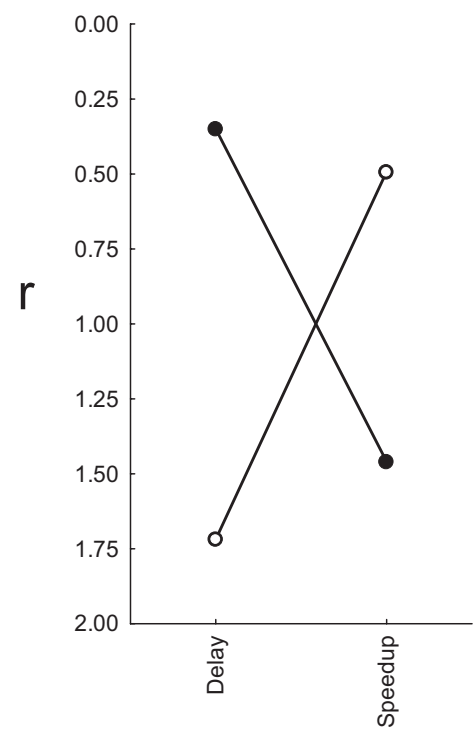

Short delay

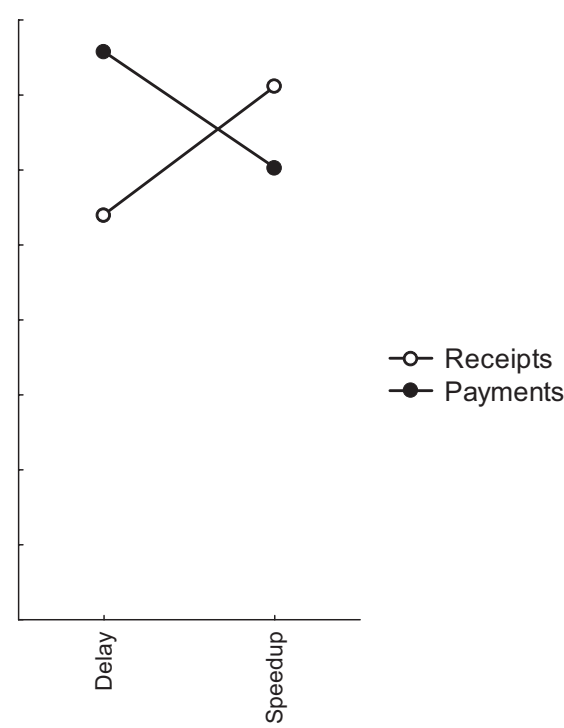

Long delay

Figure 1. Hypothetical values of $r$ (reversely scaled), showing the interaction between the delay-speedup asymmetry and delay length, more specifically, an attenuation of the delay-speedup asymmetry for longer delays. These hypothetical values also show the gain-loss asymmetry. In the bottom panels, the horizontal axis represents a discrete variable and, therefore, does not imply interpolation.

this model, loss aversion accounts for the gain-loss asymmetry, because the value difference between paying $\$ 100$ and paying $\$ 150$ is greater than the value difference between receiving $\$ 150$ and receiving \$100. Augmenting proportional sensitivity to outcomes accounts for the absolute magnitude effect, because the value difference between $\$ 1,500$ and $\$ 1,000$ is greater than the value difference between $\$ 150$ and $\$ 100$. Diminishing absolute sensitivity to delays, which is equivalent to hyperbolic discounting, accounts for the delay effect, because the weight of a delay is less than twice the weight of half the delay, or, more generally,
$w\left(t_{L}\right)<n w\left(t_{L} / n\right)$, so that a long delay $\left(t_{L}\right)$ has proportionately less weight than a short one $\left(t_{L} / n\right) .{ }^{1}$ Because the marginal impact of a

${ }^{1}$ Given a discount function $d$, hyperbolic discounting is that there is more discounting over an interval $0 \rightarrow t / 2$ than over an interval $t / 2 \rightarrow t$, that is, $d(t / 2) / d(0)<d(t) / d(t / 2)$. Given Loewenstein and Prelec's (1992) generalized hyperbolic discount function $d(t)=(1+k t)^{-b / k}$, where $b>0$ is discounting and $k>0$ is the departure from exponential discounting, $\log [d(t / 2) / d(0)]<\log [d(t) / d(t / 2)]$ yields $\log (1+k t)<2 \times \log (1+k t / 2)$, which is diminishing absolute sensitivity to delays. 
delay decreases with its length, so does the marginal impact of any change in outcome timing, which accounts for the attenuation of the delay-speedup asymmetry for longer delays. But what drives the delay-speedup asymmetry itself? This question is the focus of the next two sections, in which we develop two alternative explanations of this anomaly.

\section{Outcome Framing}

In this section, we first present the current explanation of the delay-speedup asymmetry, which ascribes the anomaly to outcome framing. We then identify two problems with it: One related to its implications, and the other related to its substance. Finally, we develop a version of the tradeoff model that continues to ascribe the delay-speedup asymmetry to outcome framing, but resolves the problems with the current explanation.

\section{The Hyperbolic Discounting Model: Reference Dependence and Loss Aversion}

Loewenstein and Prelec (1992) proposed outcome framing as an explanation for the delay-speedup asymmetry. In their hyperbolic discounting model, outcomes are evaluated as gains and losses relative to a neutral reference point. In standard choices between $S S$ and $L L$, which we call the neutral frame, the reference point is located at the status quo, so that gains and losses coincide with the actual amounts to be received or paid. In a delay or speedup frame, however, one outcome is the expected outcome whereas the other outcome compensates for foregoing the expected outcome. Obtaining a receipt or incurring a payment when expected does not carry any value, because it was expected. Foregoing the receipt or payment carries value of opposite hedonic sign: Not obtaining a receipt when expected is a loss, whereas not incurring a payment when expected is a gain. The compensating outcome, which is received or paid at a different time, is a gain or loss at that time.

Suppose one is indifferent between $S S$ and $L L$ in the neutral frame. In Loewenstein and Prelec's (1992) hyperbolic discounting model, this means that the value of $S S$ is equal to the discounted value of $L L$ :

$$
v\left(x_{S}\right)=d\left(t_{L}\right) v\left(x_{L}\right)
$$

or

$$
-v\left(x_{S}\right)+d\left(t_{L}\right) v\left(x_{L}\right)=0,
$$

where $v$ is a value function and $d$ is a generalized hyperbolic discount function. In the delay frame, not obtaining an immediate receipt is a loss that is to be compensated by a future receipt, evaluated as a gain, and not incurring an immediate payment is a gain that is to be compensated by a future payment, evaluated as a loss. One will then be indifferent between delaying and not delaying when there is full compensation:

$$
v\left(-x_{S}\right)+d\left(t_{L}\right) v\left(\hat{x}_{L}\right)=0,
$$

where $\hat{x}_{L}$ fully compensates for delaying $x_{S}$. In the absence of loss aversion, that is, $v(-x)=-v(x)$, the compensating delayed outcome $\hat{x}_{L}$ in Equation 7 is equal to the delayed outcome $x_{L}$ in Equation 6. However, in the presence of loss aversion, that is, $v(-x)>-v(x)$ for $x>0$ and $v(-x)<-v(x)$ for $x<0$, this will not be the case. For receipts, loss aversion magnifies the negative value of not obtaining the immediate receipt, so that the compensating delayed receipt must become larger. Thus, $\hat{x}_{L}>x_{L}>0$, which, in the exponential discounting model, shows up as a higher discount rate in the delay frame than in the neutral frame. For payments, loss aversion magnifies the negative value of incurring the compensating delayed payment, so that this payment must become smaller. Thus, $x_{L}<\hat{x}_{L}<0$, which, in the exponential discounting model, shows up as a lower discount rate in the delay frame than in the neutral frame. By a similar derivation for the speedup frame, we arrive at the delay-speedup asymmetry.

Loewenstein and Prelec's (1992) explanation of the delayspeedup asymmetry relies on the assumption that not obtaining an expected receipt is a loss and not incurring an expected payment is a gain, which we refer to as the symmetry assumption. As we discuss next, this assumption is not innocuous.

\section{The Symmetry Assumption}

We discuss two problems with the symmetry assumption. One is that it implies instances of negative time preference. The other is that it equates opportunity costs and out-of-pocket costs.

Negative time preference. A premise in most analyses of intertemporal choice is positive time preference: People discount delayed outcomes, so that indifference between $S S$ and $L L$ can only occur when a later outcome is larger than a sooner outcome. In the delay-speedup domain, evidence is generally consistent with this premise (e.g., Benzion et al., 1989; Malkoc \& Zauberman, 2006; McAlvanah, 2010; Shelley, 1993), even though, outside this restricted domain, positive time preference is not as common in losses as in gains (e.g., Yates \& Watts, 1975, for monetary decisions, and Ganiats et al., 2000, for health decisions). By invoking the symmetry assumption, however, Loewenstein and Prelec's (1992) hyperbolic discounting model easily arrives at predictions of negative time preference for gains as well as losses.

Predictions of negative time preference might result for the speedup of gains and the delay of losses, where loss aversion attenuates positive time preference, and potentially reverses it into negative time preference. To illustrate this for the speedup of gains, let us heuristically set the loss-aversion parameter at $\lambda=$ 2.5 , and let us further assume constant sensitivity to outcomes, that is, $v(x)=x$ (see also Thaler, Tversky, Kahneman, \& Schwartz, 1997). If, in the neutral frame, the decision maker is indifferent between receiving $\$ 100$ today and receiving $\$ 150$ in 1 year, we have, by Equation $6,-100+d(1) \times 150=0$, so that $d(1)=.67$. In the speedup frame, the decision maker would demand a minimum of $\hat{x}_{S}$ to speed up $\$ 150$ by 1 year. By Equation 7 , we have $\hat{x}_{S}+.67 \times 2.5 \times(-150)=0$, so that $\hat{x}_{S}=\$ 251.25$, an instance of negative time preference, because $\hat{x}_{S}>x_{L}$. The problem, therefore, lies in the assumption that foregoing $\$ 150$ is a full-blown loss of $\$ 150$.

No asymmetry between opportunity costs and out-of-pocket costs. An early lesson from behavioral economics is that out-ofpocket costs loom larger than opportunity costs. Thaler (1980) described the case of a man who would refuse to mow his neighbor's lawn for $\$ 20$ and yet mows his own same-sized lawn, even though his neighbor's son would be willing to mow it for only $\$ 8$. In this example, paying $\$ 8$ feels worse than not obtaining $\$ 20$, but, more generally, the asymmetry between out-of-pocket costs and 
opportunity costs is that paying $x$ feels worse than not receiving $x$. The symmetry assumption, however, asserts that not obtaining an expected receipt is a loss, meaning that not receiving $x$ as expected feels as bad as paying $x$. The anomalous prediction of negative time preference suggests that this assertion is too strong: Even foregoing an expected receipt does not have the same impact as a loss, where unfulfilled expectation would presumably intensify the sense of loss. We next develop a version of the tradeoff model that, like Loewenstein and Prelec's (1992) hyperbolic discounting model, ascribes the delay-speedup asymmetry to outcome framing, but relaxes the symmetry assumption.

\section{The Tradeoff Model: Hypersensitivity and Hyposensitivity to Worse and Better Than Expected States}

The basic tradeoff model (Equation 5) does not distinguish between outcomes on the basis of prior expectations. The value of an outcome is $v(x)$ and the value of an outcome foregone is $-v(x)$, meaning that an outcome has the same impact as an outcome foregone, that is, $v(x)-v(x)=0$, and so there will be no delay-speedup asymmetry.

To accommodate the delay-speedup asymmetry, the tradeoff model may incorporate outcome framing. Our proposal is that foregoing an expected outcome will have more or less impact than an expected outcome, depending on the sign of the outcome. On the one hand, foregoing an expected receipt is a worse than expected state, which has more impact than an unexpected receipt. This is hypersensitivity to worse than expected states. On the other hand, foregoing an expected payment is a better than expected state, which has less impact than an unexpected payment. This is hyposensitivity to better than expected states.

We can state this formally. The impact of an unexpected receipt is $v(x)$, a standard gain. The impact of an expected receipt foregone is $-\mu_{l} v(x)$, where $\mu_{l}>1$ is hypersensitivity to worse than expected states, and the subscript $l$ indicates that the expected receipt foregone is a loss. The expected but foregone receipt has therefore more impact than the unexpected but obtained one. Hypersensitivity decreases the outcome advantage of delaying a receipt, $v\left(\hat{x}_{L}\right)-\mu_{1} v\left(x_{S}\right)$, and increases the outcome disadvantage of speeding it up, $\mu_{l} v\left(x_{L}\right)-v\left(\hat{x}_{S}\right)$. To maintain indifference toward the change in outcome timing, $\hat{x}_{L}$ must become larger in the delay frame, and $\hat{x}_{S}$ must become larger in the speedup frame. The increase in $\hat{x}_{L}$ will show up as a higher discount rate, the increase in $\hat{x}_{S}$ as a lower one. That is, there will be more discounting in the delay frame than in the speedup frame, which is the delay-speedup asymmetry for receipts.

The situation for payments is the reflection of that for receipts. The impact of an unexpected payment is $v(x)$, a standard loss. The impact of an expected payment foregone is $-\mu_{g} v(x)$, where $\mu_{g}<$ 1 is hyposensitivity to better than expected states, and the subscript $g$ indicates that the expected payment foregone is a gain. The expected but foregone payment has therefore less impact than the unexpected but incurred one. Hyposensitivity increases the outcome disadvantage of delaying a payment, $\mu_{g} v\left(x_{S}\right)-v\left(\hat{x}_{L}\right)$, and decreases the outcome advantage of speeding it up, $v\left(\hat{x}_{S}\right)-\mu_{g} v\left(x_{L}\right)$. To maintain indifference toward the change in outcome timing, $\hat{x}_{L}$ must become smaller in the delay frame, and $\hat{x}_{S}$ must become smaller in the speedup frame. The decrease in $\hat{x}_{L}$ will show up as a lower discount rate, the decrease in $\hat{x}_{S}$ as a higher one. That is, there will be less discounting in the delay frame than in the speedup frame, which is the delayspeedup asymmetry for payments.

The above development of the tradeoff model ascribes the delay-speedup asymmetry to outcome framing, and is therefore referred to as the outcome-frame tradeoff model. In this model, the decision maker will be indifferent toward a change in outcome timing when:

$$
\kappa w\left(t_{L}\right)= \begin{cases}v\left(\hat{x}_{L}\right)-\mu_{l} v\left(x_{S}\right) & \text { (delaying a receipt) } \\ \mu_{l} v\left(x_{L}\right)-v\left(\hat{x}_{S}\right) & \text { (speeding up a receipt) } \\ \mu_{g} v\left(x_{S}\right)-v\left(\hat{x}_{L}\right) & \text { (delaying a payment) } \\ v\left(\hat{x}_{S}\right)-\mu_{g} v\left(x_{L}\right) & \text { (speeding up a payment), }\end{cases}
$$

where $\mu_{l}>1$ is hypersensitivity to worse than expected states, and $\mu_{g}<1$ is hyposensitivity to better than expected states. When neither outcome, $x_{S}$ or $x_{L}$, is expected, so that $\mu_{l}=\mu_{g}=1$, the outcome-frame tradeoff model (Equation 8) reduces to the basic tradeoff model (Equation 5), and predicts a full-blown gain-loss asymmetry and no delay-speedup asymmetry. When, as under the symmetry assumption, an expected receipt foregone has the same impact as a payment, that is, $\mu_{l}=\lambda$, and an expected payment foregone has the same impact as a receipt, that is, $\mu_{g}=1 / \lambda$, the model predicts a full-blown delay-speedup asymmetry and no gain-loss asymmetry. Finally, when $1 / \lambda<\mu_{g}<1<\mu_{l}<\lambda$, it predicts both asymmetries. The less the values of $\mu_{l}$ and $\mu_{g}$ depart from their neutral value of 1 , the less likely it becomes that the outcome-frame tradeoff model yields negative time preference.

While the outcome-frame tradeoff model qualitatively predicts all anomalies under consideration, we next achieve the same by ascribing the delay-speedup asymmetry to time framing rather than outcome framing.

\section{Time Framing}

Let us look again at the basic phenomenon. Suppose you expect the delivery of a parcel today, but you are told that it can only be delivered next month. How much would you have to be paid to feel you are being fully compensated by the company for this delay? Call this $C$. Now suppose you expect the delivery of a parcel next month, but you are told that it can already be delivered today. How much would you pay to feel you are fully compensating the company for this speedup? Call this $c$. The delay-speedup asymmetry is that $C>c$, but what is driving this effect?

According to the outcome-frame tradeoff model, it is because, in both scenarios, you are in a worse than expected state when not receiving the parcel when expected. This drives up the price you demand in the delay frame, but drives down the price you offer in the speedup frame. It seems a plausible explanation for the delay frame, where you receive the desired parcel later rather than sooner. It is less plausible, however, for the speedup frame, where you receive the desired parcel sooner rather than later: Why be frustrated when something good is happening to you sooner than expected? In general, it seems plausible that one would be frustrated when something good comes later than expected and be relieved when something bad comes later than expected, but it is implausible that one would be frustrated when something good comes sooner than expected and be relieved when something bad comes sooner than expected. 
When we change from outcome framing to time framing, we arrive at a more plausible explanation of the delay-speedup asymmetry. In the delay frame, something good is happening later rather than sooner, which is treated as a loss of time, and hypersensitivity to time lost drives up the price you demand. In the speedup frame, something good is happening sooner rather than later, which is treated as a gain of time, and hyposensitivity to time gained drives down the price you offer. The converse occurs when something bad will be happening, like being laid off. If the notification says you will go tomorrow, you would be willing to pay for delaying the lay-off. That is, you would pay for something bad to happen later rather than sooner, which is treated as a gain of time. Hyposensitivity to time gained drives down the price you would offer for the delay. If the notification says you will go 6 months from now, you would want to be paid for speeding up the lay-off. That is, you would be paid for something bad to happen sooner rather than later, which is treated as a loss of time. ${ }^{2}$ Hypersensitivity to time lost drives up the price you would demand for the speedup. It must be emphasized that no time is "gained" or "lost" in and of itself; rather, time is gained or lost in obtaining something good or incurring something bad.

More formally, the time disadvantage of delaying a receipt or speeding up a payment is $\kappa_{l} \kappa w\left(t_{L}\right)$, where $\kappa>0$ is the tradeoff parameter from Equation 5. Hypersensitivity to time lost, that is, $\kappa_{l}>1$, increases this time disadvantage. Thus, to maintain indifference toward the change in outcome timing, $\hat{x}_{L}$ must become larger when delaying a receipt, and $\hat{x}_{S}$ must become smaller when speeding up a payment, which will show up as a higher discount rate. On the other hand, the time advantage of speeding up a gain or delaying a loss is $\kappa_{g} \kappa w\left(t_{L}\right)$. Hyposensitivity to time gained, that is, $0<\kappa_{g}<1$, decreases this time advantage. Thus, to maintain indifference toward the change in outcome timing, $\hat{x}_{S}$ must become larger in speeding up a receipt, and $\hat{x}_{L}$ must become smaller in delaying a payment, which will show up as a lower discount rate. Altogether, there will be more discounting when delaying a receipt than when speeding it up, and less discounting when delaying a payment than when speeding it up, which is the delay-speedup asymmetry.

The above development of the tradeoff model ascribes the delay-speedup asymmetry to time framing, and is therefore referred to as the time-frame tradeoff model. In this model, the decision maker will be indifferent toward a change in outcome timing when:

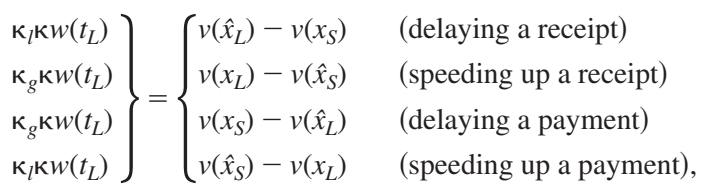

where $\kappa_{l}>1$ is hypersensitivity to time lost, and $0<\kappa_{g}<1$ is hyposensitivity to time gained. The time-frame tradeoff model (Equation 9) reduces to the basic tradeoff model (Equation 5) when neither outcome is expected, so that $\kappa_{g}=\kappa_{l}=1$.

In addition to the delay-speedup asymmetry, the time-frame tradeoff model predicts the gain-loss asymmetry (loss aversion), the absolute magnitude effect (augmenting proportional sensitivity to outcomes), and both the delay effect and an attenuation of the delay-speedup asymmetry for longer delays (diminishing absolute sensitivity to delays). The outcome-frame tradeoff model yields the same qualitative predictions. Given that the models produce the same qualitative predictions, our purpose is to compare the models on their quantitative predictions. The exponential discounting model, which yields none of the above predictions, will serve as a benchmark to evaluate the predictive accuracy of the tradeoff models. Because the exponential discounting model may be viewed as an overly naïve benchmark model, however, we next turn to some more informed models that we will consider in our model contest.

\section{Simple Interest Rates and Hyperbolic Discount Rates}

Extremely popular in research on intertemporal choice is the notion of hyperbolic discounting, which, as Prelec (2004) observed, "now functions almost as a default option for analyzing the misbehavior of economic agents" (p. 511). So we must include that default option in our analyses. The notion of hyperbolic discounting emerges from a model in which the person will be indifferent between $S S$ and $L L$ when:

$$
x_{S}\left(1+k t_{L}\right)=x_{L} .
$$

In this model, the person demands simple interest: Per unit of time, the interest demanded is $k x_{S}$, meaning that interest does not bear interest. The simple-interest model accommodates the delay effect. To illustrate, someone indifferent between $\$ 100$ today and $\$ 150$ in 1 year might also be indifferent between $\$ 100$ today and $\$ 200$ in 2 years. The simple interest rate is $50 \%$ in each case, but the compound interest rate is $50 \%$ in the first case and $41 \%$ in the second, which is the delay effect. The simple-interest model describes present-to-future conversion, but it may be changed so as to describe future-to-present conversion instead, which is what the hyperbolic discounting model does:

$$
x_{S}=\frac{1}{1+k t_{L}} x_{L},
$$

where $1 /\left(1+k t_{L}\right)$ is the hyperbolic discount function proposed by Mazur (1987). Under hyperbolic discounting, there is not a single, constant discount rate. Rather, the discount rate decreases with the delay to the outcome. Specifically, over the per-period interval $t-1 \rightarrow t$, the discount rate is:

$$
\rho_{t}=\frac{k}{1+k t},
$$

which decreases with delay length $t$. The hyperbolic discounting model in Equation 10 and the exponential discounting model in Equation 3 are both special cases of a model that includes the generalized hyperbolic discount function proposed by Loewenstein and Prelec (1992):

$$
x_{S}=\frac{1}{\left(1+k t_{L}\right)^{b / k}} x_{L},
$$

where $b>0$ is discounting and $k>0$ is the departure from exponential discounting. This generalized hyperbolic discounting

\footnotetext{
${ }^{2}$ This is even more obvious in strictly monetary tradeoffs: If you speed up a payment, you can think of it as a loss of profit from investment or as a loss of time during which the money could have been invested.
} 
model reduces to hyperbolic discounting model when $b=k$ and to the exponential discounting model when $k$ goes toward zero, in which case the discount function becomes $e^{-b t_{L}}=\delta^{t_{L}}$. The hyperbolic discounting model and the generalized hyperbolic discounting model are widely used in empirical research on intertemporal choice, so that they are suitable benchmark models. However, they only accommodate the delay effect. Considering that our focal anomaly is the delay-speedup asymmetry, we next incorporate this anomaly as well into the generalized hyperbolic discounting model.

\section{Outcome Framing Revisited}

In the section on outcome framing, we discussed how Loewenstein and Prelec's (1992) hyperbolic discounting model explains the delay-speedup asymmetry. The two ingredients were reference dependence and loss aversion. We incorporate that explanation into the generalized hyperbolic discounting model. When delaying a receipt, the immediate receipt foregone is a loss, to be compensated by a delayed receipt, which is a gain. Conversely, when delaying a payment, the immediate payment foregone is a gain, to be compensated by a delayed payment, which is a loss. The same rationale applies when a receipt or a payment is expedited. The decision maker will be indifferent toward changes in outcome timing when:

$$
0= \begin{cases}-\lambda x_{S}+d\left(t_{L}\right) \hat{x}_{L} & \text { (delaying a receipt) } \\ \hat{x}_{S}-d\left(t_{L}\right) \lambda x_{L} & (\text { speeding up a receipt) } \\ -x_{S}+d\left(t_{L}\right) \lambda \hat{x}_{L} & \text { (delaying a payment) } \\ \lambda \hat{x}_{S}-d\left(t_{L}\right) x_{L} & \text { (speeding up a payment) }\end{cases}
$$

where $\lambda>1$ is loss aversion, and $d$ is the generalized hyperbolic discount function from Equation 11. The above discounting model ascribes the delay-speedup asymmetry to outcome framing, and is therefore referred to as the outcome-frame discounting model.

The outcome-frame discounting model accommodates all three time-related anomalies: The delay-speedup asymmetry, the delay effect, and an attenuation of the delay-speedup asymmetry for longer delays. However, it does not cover the two outcome-related anomalies: The gain-loss asymmetry and the absolute magnitude effect. This is because the value function of the outcome-frame discounting model exhibits constant sensitivity, where the value function of Loewenstein and Prelec's (1992) hyperbolic discounting model exhibits diminishing absolute sensitivity and, to account for the gain-loss asymmetry and the absolute magnitude effect, two technical properties related to the elasticity of the value function. ${ }^{3}$ Parametric specifications of such a value function are inevitably complex (al-Nowaihi \& Dhami, 2009; Scholten \& Read, 2012), and there are still the problems, identified in the sections on outcome framing and time framing, with the way in which Loewenstein and Prelec's (1992) hyperbolic discounting model explains the delay-speedup asymmetry. We therefore do not further develop the benchmark models beyond the outcome-frame discounting model.

\section{Three Experimental Tests}

We conducted three tests of the competing models. First, we applied the models to the indifference data from the matching study reported by Benzion et al. (1989). Because the design used by these authors did not include the neutral frame, we subsequently applied the models to the indifference data from a new matching study that did include the neutral frame. Finally, we applied the models to preference data from a new choice study. We offer the first integrative analysis of indifference and preference in the neutral, delay, and speedup frames, and the first quantitative application of psychological choice models to data containing the delay-speedup asymmetry.

\section{Indifference Data From Matching: A Reanalysis of Benzion et al. (1989)}

\section{Method}

Independent and dependent variables. For 64 option pairs, participants filled in the variable outcome $\hat{x}$ that yielded indifference between $S S$ and $L L$. A concrete scenario designated this variable outcome as a compensation for delaying or speeding up the fixed outcome $x$. The 64 option pairs resulted from a 2 (decision frame: delay or speedup) $\times 2$ (outcome sign: positive or negative) $\times 4$ (outcome magnitude: $\$ 40, \$ 200, \$ 1,000$, or $\$ 5,000) \times 4$ (delay to the larger outcome: $1 / 2$ year, 1 year, 2 years, or 4 years) factorial design. Benzion et al. (1989) reported the arithmetic means of the compound interest rates $r$, as given by Equation 2. Although this practice is common (e.g., Malkoc \& Zauberman, 2006; Shelley, 1993), it is flawed because taking the arithmetic mean of the interest rate $r$ is not the same as taking the arithmetic means of $x_{S}$ and $x_{L}$ and computing $r$ from there (cf. Kirby, 1997), meaning the functional relation between outcomes and discount measures is not preserved in the aggregate data.

Model specification. Our theoretical introduction exhaustively specified the competing models, except for the value function and the time-weighing function of the tradeoff models. As proposed originally (Scholten \& Read, 2010), these are specified as normalized logarithmic functions. The value function is:

$$
v(x)=\left\{\begin{array}{l}
\frac{1}{\gamma} \log (1+\gamma x) \text { if } x \geq 0 \\
-\frac{\lambda}{\gamma} \log (1+\gamma(-x)) \text { if } x<0,
\end{array}\right.
$$

where $\gamma>0$ is diminishing absolute sensitivity to outcomes and $\lambda>1$ is constant loss aversion. As $\gamma$ goes to zero, the value function exhibits constant sensitivity, that is, $v(x)=x$ for $x>0$ and $v(x)=\lambda x$ for $x<0$, and, as $\gamma$ goes to infinity, it exhibits insensitivity, that is, $v(x)=0$. The time-weighing function is:

$$
w(t)=\frac{1}{\tau} \log (1+\tau t),
$$

where $\tau>0$ is diminishing absolute sensitivity to delays. The limiting conditions are the same as those for the value function, with diminishing sensitivity bordering on constant sensitivity when $\tau$ goes to zero and insensitivity when $\tau$ goes to infinity.

\footnotetext{
${ }^{3}$ Theoretical analyses of the delay-speedup asymmetry have typically invoked constant sensitivity (Loewenstein, 1988; Scholten \& Read, 2010; Shelley, 1993).
} 
Model estimation and evaluation. We estimated seven models: Three tradeoff models (basic, outcome-frame, and time-frame) and four discounting models (exponential, hyperbolic, generalized hyperbolic, and outcome-frame). The exponential discounting model predicts a constant value of $r$, all other models predict that it will vary. To estimate the models, we solved the equations of the three tradeoff models $(5,8$, and 9$)$ and the four discounting models $(3,10,11$, and 12$)$ for the variable outcome $\hat{x}$, and inserted it, along with the fixed outcome $x$ and the delay $t_{L}$, into Equation 2 to obtain the predicted value of $r$. We estimated the models with the HookeJeeves and quasi-Newton routine in the nonlinear estimation module of Statistica (StatSoft, 2003) by minimizing the sum of squared deviations between the predicted and observed values of $r$. Given this loss function, the exponential discounting model predicts the mean value of $r$.

In comparing our models, we must take into account that data are noisy, and that a more complex model is more flexible in capturing the noise (Myung, Tang, \& Pitt, 2009). That is, a more complex model in terms of number of parameters and the functional form (i.e., the way in which the parameters and the variables are combined in the model equation) can come out best from the model comparison because it is more flexible in capturing the noise, and not because it best describes the mental process (Pitt, Myung, \& Zhang, 2002). Because the noise will change from one data set to the other, while the regularity from the mental process will not, the logical thing to do is to estimate a model on one data set, and evaluate it on another; the more noise a model captures in the estimation phase, the worse it will fit in the evaluation phase, and, conversely, the more regularity the model captures in the estimation phase, the better is will fit in the evaluation phase (Myung et al., 2009). Therefore, we must change from goodness-of-fit to generalizability as criterion for model comparison. Among available methods, crossvalidation emerges as an easy-to use, heuristic method for assessing generalizability (Pitt et al., 2002). We use $n$-fold cross-validation, or the leave-one-out method, in which each data point is predicted upon estimating the model on the remaining $n-1$ data points (Efron \& Tibshirani, 1993). In this reanalysis of Benzion et al. (1989), $n=64$. The models are compared on their badness-of-fit, that is, the deviation between observations and the predictions thus obtained.

\section{Results}

The results showed all preference patterns under study: The delay-speedup asymmetry, the gain-loss asymmetry, the absolute magnitude effect, and the delay effect. Figure 2 shows the observed values of $r$, omitting outcome magnitude. (The values of $r$ are reversely scaled for comparability with subsequent results.) We see the delay-speedup asymmetry, and, consistent with Figure 1, its attenuation for longer delays. We further see the gain-loss asymmetry for all delays, except for 2 years. This is an anomalous result that the tradeoff models will be unable to explain

Table 1 shows the number of free parameters and the badness-of-fit of the seven models. Because the design of Benzion et al. (1989) does not include the neutral frame, the time-frame tradeoff model cannot estimate both hypersensitivity to time lost and hyposensitivity to time gained, so that it has one parameter less than the outcome-frame tradeoff model. We estimated hypersensitivity, but we would obtain exactly the same results if we estimated hyposensitivity instead. Going down the list of competing models, badness-of-fit decreases, with the time-frame tradeoff model coming out best.

The outcome-frame and time-frame tradeoff models are the only ones that qualitatively predict the full range of anomalies in these data. Table 2 shows the parameter estimates upon estimating these models on the set of $n$ data points. There is

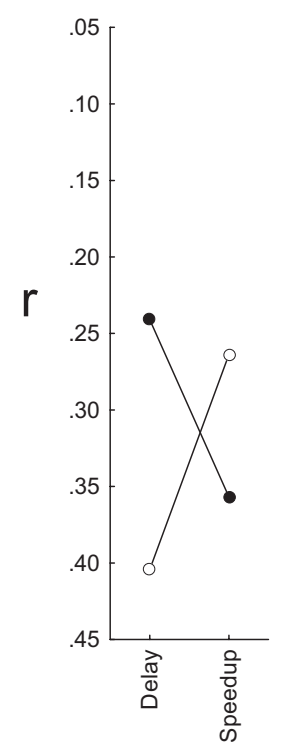

$1 / 2$ year

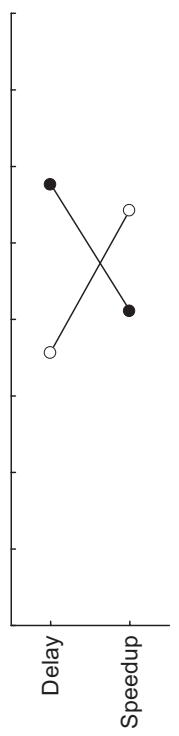

1 year

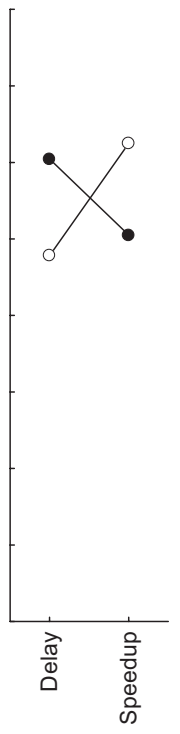

2 years

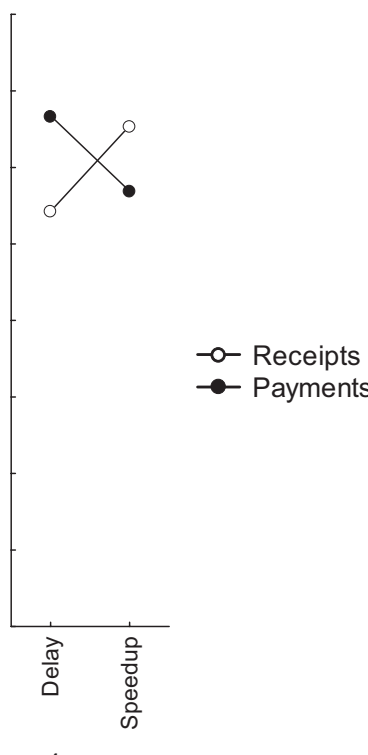

4 years

Figure 2. Benzion et al.'s (1989) indifference data from matching. This figure shows the observed values of $r$ (reversely scaled). We observe the delay-speedup asymmetry and its attenuation for longer delays. 
Table 1

Benzion et al.'s (1989) Indifference Data From Matching: Badness-of-Fit Upon Cross-Validating the Discounting Models and the Tradeoff Models on $r$

\begin{tabular}{llrr}
\hline \multicolumn{1}{c}{ Model } & $k^{\mathrm{a}}$ & $\mathrm{RMSD}^{\mathrm{b}}$ & $-\log (L)^{\mathrm{c}}$ \\
\hline Exponential discounting model & 1 & 0.10 & -54.57 \\
Hyperbolic discounting model & 1 & 0.09 & -62.18 \\
Generalized hyperbolic discounting model & 2 & 0.08 & -67.94 \\
Outcome-frame discounting model & 3 & 0.07 & -75.12 \\
Basic tradeoff model & 4 & 0.07 & -80.66 \\
Outcome-frame tradeoff model & 6 & 0.06 & -86.90 \\
Time-frame tradeoff model & 5 & 0.04 & -108.40 \\
\hline
\end{tabular}

${ }^{\mathrm{a}}$ Number of free parameters. ${ }^{\mathrm{b}}$ The root-mean-square deviation, $\sqrt{\sum\left(y_{i}-\hat{y}_{i}^{-i}\right)^{2} / n}$, where $n$ is the number of data points, $y_{i}$ is the observed value of dependent variable $y$ for data point $i$, and $\hat{y}_{i}^{-i}$ is the predicted value of $y$ for data point $i$, obtained by estimating the model on the remaining $n-1$ data points. The standard deviation, $\sqrt{\sum\left(y_{i}-\bar{y}\right)^{2} / n}$, where $\bar{y}$ is the mean value of $y$, is $0.10 .{ }^{\mathrm{c}}-\log (L)=0.5 n\left(\log \left(\sum\left(y_{i}-\hat{y}_{i}^{-i}\right)^{2} / n\right)+\log (2 \pi)+1\right)$, where $\log (L)$ is the maximum log-likelihood of the estimated model (see Burnham \& Anderson, 2003; Spiess \& Neumeyer, 2010).

modest loss aversion $(\lambda)$, reflecting a modest gain-loss asymmetry in the data. The time-frame tradeoff model exhibits hypersensitivity to time lost $\left(\kappa_{l}\right)$, and the outcome-frame tradeoff model exhibits hypersensitivity to worse than expected states $\left(\mu_{l}\right)$ and hyposensitivity to better than expected states $\left(\mu_{g}\right)$.

The top and bottom panels of Figure 3 show the predictions of the outcome-frame tradeoff model and the time-frame tradeoff model, respectively. It is clear that the time-frame tradeoff model most closely reproduces the qualitative patterns in the data. Although both models predict the delay-speedup and gain-loss asymmetries, and their attenuation for longer delays, the outcome-frame tradeoff model predicts far too much attenuation, so much so that, at the 4-year delay, it predicts no delay-speedup asymmetry for gains.

Figure 4 shows the results for outcome magnitude. The models yield the same predictions, involving progressively smaller decreases of $r$ as outcome magnitude increases. The result for the $\$ 5,000$ outcomes, showing a relatively large decrease of $r$, is anomalous to this prediction, and it suggests that outcomes of this magnitude were qualitatively different for the participants.

\section{More Indifference Data From Matching}

In this study, we analyze indifference data from a matching study that includes the neutral frame, so that we can estimate the full time-frame tradeoff model, and evaluate the efficacy of this model and the outcome-frame tradeoff model in predicting the gain-loss asymmetry in both the neutral frame and the delay-speedup frames.

\section{Method}

Independent and dependent variables. There were 32 option pairs resulting from a 2 (outcome matched: $x_{L}$ or $\left.x_{S}\right) \times 2$ (decision frame: neutral or delay-speedup) $\times 2$ (outcome sign: positive or negative) $\times 2$ (outcome magnitude: $€ 100$ or $€ 900) \times 2$ (delay to the larger outcome: $1 / 2$ year or 2 years) factorial design. The first two factors were manipulated between participants, the others within participant, so that each participant matched eight options pairs. For each option pair, we computed the geometric mean of the per-period discount factor $\delta$ (Equation 4). This practice is to be recommended, because taking the geometric mean of discount factor $\delta$ is the same as taking the geometric means of $x_{S}$ and $x_{L}$ and computing $\delta$ from there, meaning the functional relation between outcomes and discount measures is preserved in the aggregate data. Analyses were performed on arithmetic means of $\log (\delta)$. The full breakdown of our results is given in Table $3 .^{4}$

Participants. A total of 280 Portuguese residents, $45 \%$ male and averaging 33 years of age, participated by completing an on-line questionnaire. Invitations for participating in the investigation were sent out by e-mail to the acquaintances of eight students who had enrolled in a research seminar of the first author. The receivers of this e-mail were urged to invite their acquaintances in turn. Most participants had an academic degree (84\%), and most were employed $(59 \%)$ or students $(34 \%)$.

Procedure. Participants filled in the variable outcome that yielded indifference between $S S$ and $L L$. An item for the delay of a receipt would read: "You are entitled to receive $€ 100$ today, but you can choose to delay the receipt, and receive more in 2 years." The participants then filled in the blank in the following statement: "Instead of receiving $€ 100$ today, I agree to delay the receipt if I

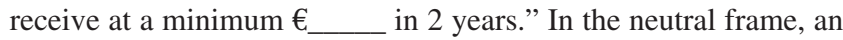
item for the $x_{L}$ matching of a receipt would read: "For me, receiving $€ 100$ today is as good as receiving €__ in 2 years" (cf. Takeuchi, 2011). ${ }^{5}$ Similar items were developed for the re-

\footnotetext{
${ }^{4}$ Ninety participants discounted an outcome by a greater amount over the $1 / 2$ year interval than over the 2 years interval for at least one of the option pairs in the outcome sign $\times$ outcome magnitude subdesign, or discounted the $\$ 100$ outcome by a greater amount than the $\$ 900$ outcome for at least one of the option pairs in the outcome sign $\times$ delay length subdesign. They were excluded from the analyses. The $68 \%$ survival rate (190/280) is comparable to the $72 \%$ survival rate (204/282) reported by Benzion et al. (1989), but their criterion was incomplete rather than incorrect responding (but see Shelley, 1993; Thaler, 1981). The survival rates were significantly higher for $x_{L}$ matching than for $x_{S}$ matching, $.81-$ $.54=.27, \chi^{2}(1)=23.64, p=.00$, which suggests that our participants had far less difficulty with present-to-future than with future-to-present conversions. The exclusion of inconsistent responders may not be innocuous, in that their mental process may not be the same as that of the consistent responders.

${ }^{5}$ Shelley (1993, Figure 2, p. 811) operationalized the neutral frame as follows: "You owe a debt of $\$ x$ in $t$ years to a public institute. What is the (negative) value, $-\$ x$, of that debt to you now?" Her results suggest that this was understood as a speedup frame instead: Implied discount rates in the "neutral" frame were higher for losses than for gains, just as they were in the speedup frame.
} 
Table 2

Benzion et al.'s (1989) Indifference Data From Matching: Parameter Estimates and Statistical Tests Upon Estimating the Tradeoff Models on $r$

\begin{tabular}{|c|c|c|c|c|c|c|c|}
\hline \multicolumn{4}{|c|}{ Outcome framing } & \multicolumn{4}{|c|}{ Time framing } \\
\hline Parameter & Estimate & $t(58)^{\mathrm{a}}$ & $p$ & Parameter & Estimate & $t(59)^{\mathrm{a}}$ & $p$ \\
\hline \multirow[t]{2}{*}{$\kappa$} & 7.53 & 6.06 & .00 & $\kappa$ & 6.39 & 7.93 & .00 \\
\hline & & & & $\kappa_{l}$ & 1.45 & 7.25 & .00 \\
\hline$\tau$ & 1.75 & 3.68 & .00 & $\tau$ & 1.77 & 4.91 & .00 \\
\hline$\gamma$ & 0.04 & 6.54 & .00 & $\gamma$ & 0.04 & 8.84 & .00 \\
\hline$\lambda$ & 1.07 & 1.31 & .10 & $\lambda$ & 1.13 & 2.88 & .00 \\
\hline$\mu_{l}$ & 1.01 & 3.87 & .00 & & & & \\
\hline$\mu_{g}$ & 0.99 & -3.25 & .00 & & & & \\
\hline
\end{tabular}

Note. The models were estimated on 64 data points (see Benzion et al., 1989, Table 1).

${ }^{a}$ Testing whether $\kappa, \tau$, and $\gamma$ are reliably greater than zero, whether $\kappa_{l}, \lambda$, and $\mu_{l}$ are reliably greater than one, and whether $\mu_{g}$ is reliably smaller than one.

maining conditions. In the introduction to the questionnaire, participants completed a rehearsal trial. Upon completing the experimental trials, they provided their demographics.

\section{Results}

We conducted a mixed-design analysis of variance (ANOVA) on $\log (\delta)$ with three within-participant factors (outcome sign, outcome magnitude, and delay length) and two betweenparticipants factors: One factor contrasted $x_{L}$ matching with $x_{S}$ matching, the other factor contrasted the neutral frame with the delay-speedup frames. Seven main results emerged from this analysis: (1) The delay-speedup and gain-loss asymmetries in the delay-speedup frames and the gain-loss asymmetry in the neutral frame, $F(1,186)=21.40, p=.00, \eta_{P}^{2}=.12$; (2) the gain-loss asymmetry across frames, $F(1,186)=13.66, p=.00, \eta_{P}^{2}=.07$; (3) a less pronounced gain-loss asymmetry in the delay-speedup frames than in the neutral frame, $F(1,186)=4.22, p=.04, \eta_{P}^{2}=$ .02 ; (4) an attenuation of the delay-speedup and gain-loss asymmetries for longer delays, $F(1,186)=15.52, p=.00, \eta_{P}^{2}=.08$; (5) the absolute magnitude effect, $F(1,186)=26.97, p=.00$, $\eta_{P}^{2}=.14 ;(6)$ an attenuation of the absolute magnitude effect for longer delays, $F(1,186)=5.62, p=.02, \eta_{P}^{2}=.03$; and (7) the delay effect, $F(1,186)=81.41, p=.00, \eta_{P}^{2}=.44 .^{6}$ The left panels of Figure 5 show the observed values of $\delta$ along a logarithmic scale. In the neutral frame, $x_{S}$ matching and $x_{L}$ matching yielded virtually the same results, so that we collapse across these conditions.

Table 4 shows the number of free parameters and the badnessof-fit of the seven models upon $n$-fold cross-validation on the experimental trials. Going down the list of competing models, badness-of-fit decreases, but with one exception: The outcomeframe discounting model, which fails to predict the gain-loss asymmetry and the absolute magnitude effect, came out better than the basic tradeoff model, which fails to predict the delay-speedup asymmetry, and the outcome-frame tradeoff model, which qualitatively predicts all anomalies in the data. This suggests that the added complexity of the outcome-frame tradeoff model captured more noise than regularity. The time-frame tradeoff model comes out best from the model contest.

Table 5 shows the parameter estimates upon estimating the outcome-frame and time-frame tradeoff models on the full set of $n$ data points. Loss aversion $(\lambda)$ is more pronounced than in the reanalysis of Benzion et al. (1989). In the time-frame tradeoff model, hypersensitivity to time lost is more pronounced than hyposensitivity to time gained, that is, $\log \left(\kappa_{l}\right)=0.65>0.14=$ $-\log \left(\kappa_{g}\right){ }^{7}$ In the outcome-frame tradeoff model, however, hypersensitivity to worse than expected states is no more pronounced, and slightly less pronounced, than hyposensitivity to better than expected states, that is, $\log \left(\mu_{l}\right)=0.05<-\log \left(\mu_{g}\right)=0.06$.

The center panels and right panels of Figure 5 show the predictions of the outcome-frame tradeoff model and time-frame tradeoff model, respectively. In the predictions of both models, the gainloss asymmetry is as pronounced in the delay-speedup frames as in the neutral frame, whereas, in the data, the gain-loss asymmetry is less pronounced in the delay-speedup frames than in the neutral frame. This can be seen more easily in Figure 6. It can also be seen that, across gains and losses, the outcome-frame tradeoff model incorrectly predicts as much discounting in the delay-speedup frames as in the neutral frame, while the time-framing tradeoff model correctly predicts more discounting in the delay-speedup frames than in the neutral frame. Omitted from Figures 5 and 6 is outcome magnitude. Both tradeoff models closely reproduced the absolute magnitude effect and its attenuation for longer delays.

\footnotetext{
${ }^{6}$ Corollary and secondary results emerged from the ANOVA: (I) More discounting in the delay-speedup frames than in the neutral frame, $F(1$, 186) $=6.01, p=.02, \eta_{P}^{2}=.03$; (II) an attenuation of the difference in discounting between the delay-speedup frames and the neutral frame for longer delays, $F(1,186)=10.80, p=.00, \eta_{P}^{2}=.06$; (III) an outcome sign by outcome matched interaction across decision frames, $F(1,186)=20.37$, $p=.00, \eta_{P}^{2}=.11$ (a corollary of Result 1); (IV) an attenuation of the outcome sign by outcome matched interaction for longer delays, $F(1$, $186)=13.00, p=.00, \eta_{P}^{2}=.07$ (a corollary of Result 4); (V) an outcome sign by outcome magnitude interaction, in that the gain-loss asymmetry was more pronounced for small outcomes than for large ones, $F(1,186)=$ 7.74, $p=.01, \eta_{p}^{2}=.04$ (see Loewenstein \& Prelec, 1992); (VI) an attenuation of the outcome sign by outcome magnitude interaction for longer delays, $F(1,186)=4.12, p=.04, \eta_{P}^{2}=.02 ;(\mathrm{VII})$ an outcome magnitude by outcome matched interaction, in that the absolute magnitude effect was more pronounced when the later outcome was matched than when the sooner outcome was matched, $F(1,186)=17.16, p=.00, \eta_{P}^{2}=$ .09 ; and (VIII) an attenuation of the outcome magnitude by outcome matched interaction for longer delays, $F(1,186)=7.70, p=.01, \eta_{P}^{2}=.04$

${ }^{7}$ Because $\kappa_{l}>1$ and $0<\kappa_{g}<1$, we must compare $\log \left(\kappa_{l}\right)$ with $-\log \left(\kappa_{g}\right)$.
} 


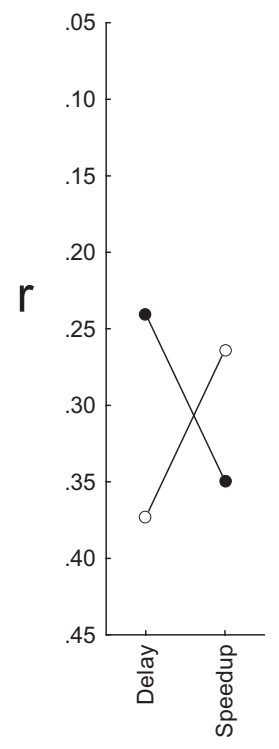

$1 / 2$ year

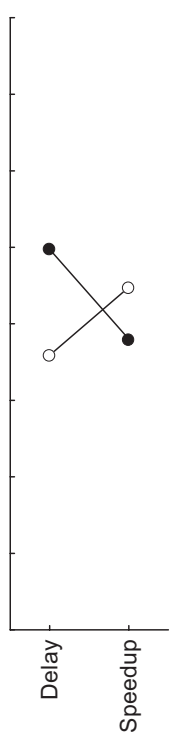

1 year

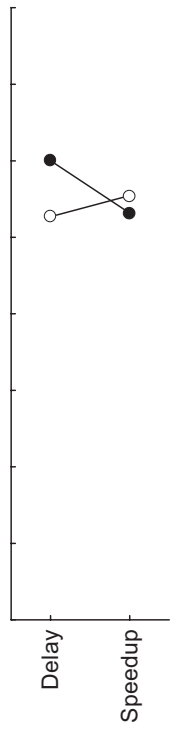

2 years

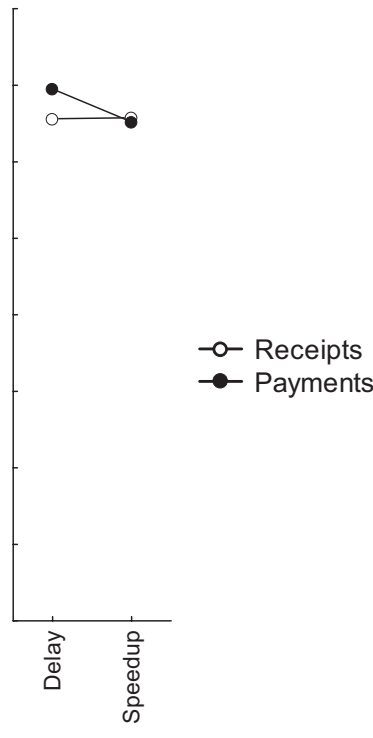

4 years

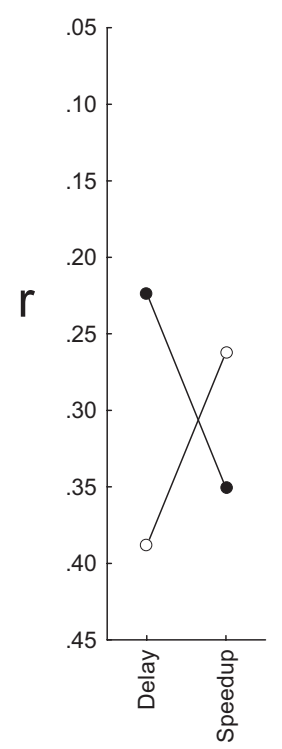

$1 / 2$ year

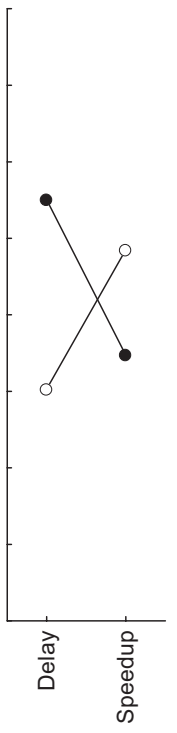

1 year

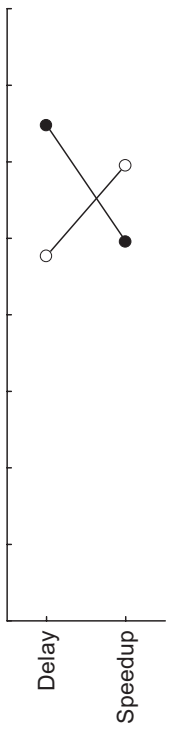

2 years

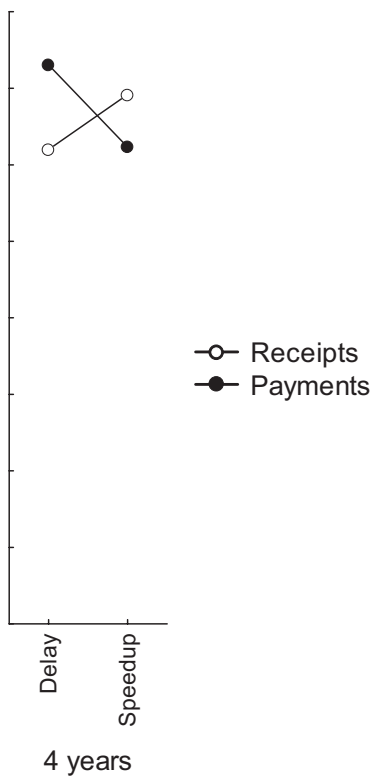

Figure 3. Benzion et al.'s (1989) indifference data from matching. This figure shows the predicted values of $r$ from the outcome-frame tradeoff model (top panel) and the time-frame tradeoff model (bottom panel).

It is possible to run an additional cross-validation check, because, in the introduction to the questionnaire, the participants performed a rehearsal trial involving a medium-large gain (€300, the geometric mean of $€ 100$ and $€ 900)$ over a medium-length interval ( 1 year, the geometric mean of $1 / 2$ year and 2 years), and this trial can be used for cross-validation. For each model, we used the parameters estimated from the 32 experimental trials to predict $\log (\delta)$ in the four different rehearsal trials corresponding to the four cells in the 2 (outcome matched) $\times 2$ (decision frame) between-participants subdesign. This subdesign only includes the delay-speedup asymmetry, and none of the remaining anomalies (i.e., the gain-loss asymmetry, the absolute magnitude effect, the delay effect, and the attenuation of the delay-speedup asymmetry for longer delays). The results are shown in Table 4, under the heading "rehearsal trial." Among the models that fail to predict the delay-speedup asymmetry, the basic tradeoff model came out worse than the three discounting models (exponential, hyperbolic, and generalized hyperbolic), and, among these discounting models, the generalized hyperbolic discounting model came out worse than the two discounting models it includes as special cases (exponential and hyperbolic), suggesting that the added complexity may be a handicap in the extrapolation to stimuli with different 


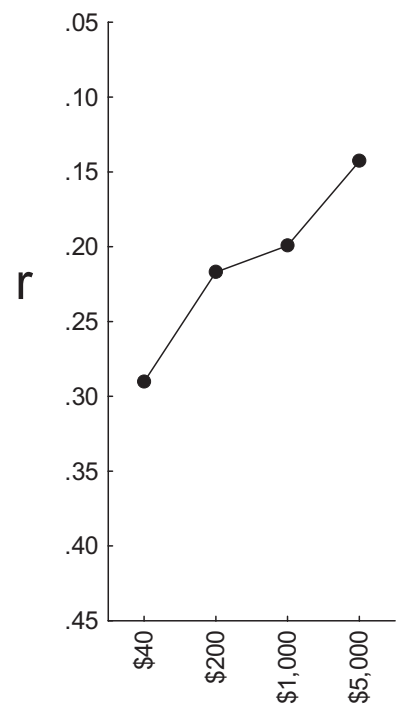

Data
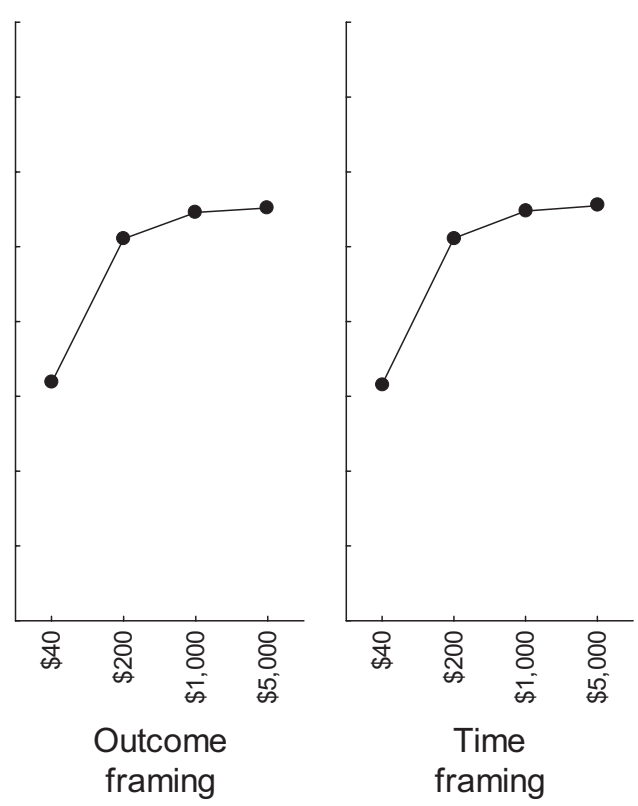

Figure 4. Benzion et al.'s (1989) indifference data from matching. Observed and predicted values of $r$ (reversely scaled). Predictions from the tradeoff models. We observe the absolute magnitude effect for $\$ 200$, its attenuation for $\$ 1,000$, and its accentuation for $\$ 5,000$.

characteristics (i.e., a constant rather than a varying outcome sign, a different and constant outcome magnitude, and a different and constant delay length). Again, the outcome-frame tradeoff model came out worse than the outcome-frame discounting model, which, in turn, came out worse than the time-frame tradeoff model, the winner in this additional cross-validation check.

\section{Discussion}

Among the models that predict the delay-speedup asymmetry, the time-frame tradeoff model came out better than the outcome- frame tradeoff model and the outcome-frame discounting model, consistent with our reanalysis of the data from Benzion et al. (1989). However, outcome-frame tradeoff model came out slightly worse than the outcome-frame discounting model, when the former is more comprehensive than the latter. This suggests that the added complexity of the outcome-frame tradeoff model captured more noise than regularity, even though it captured the gain-loss asymmetry and the absolute magnitude effect, whereas the outcome-frame discounting model did not. Actually, our own matching data may have been noisier than Benzion et al.'s data,

Table 3

Indifference Data From Matching: Delays, Outcomes, and Per-Period Discount Factors

\begin{tabular}{|c|c|c|c|c|c|c|c|}
\hline \multicolumn{4}{|c|}{ Neutral frame ${ }^{\mathrm{a}}$} & \multicolumn{4}{|c|}{ Delay-speedup frames ${ }^{\mathrm{b}}$} \\
\hline$t_{L}^{\mathrm{c}}$ & $x_{S}{ }^{\mathrm{d}}$ & $x_{L}^{\mathrm{d}}$ & $\delta^{e}$ & $t_{L}^{\mathrm{c}}$ & $x_{S}{ }^{\mathrm{d}}$ & $x_{L}{ }^{\mathrm{d}}$ & $\delta^{\mathrm{e}}$ \\
\hline $1 / 2$ & 100 & 159.45 & .393 & $1 / 2$ & 100 & 204.08 & .240 \\
\hline 2 & 100 & 264.27 & .615 & 2 & 100 & 364.67 & .524 \\
\hline $1 / 2$ & 900 & $1,054.51$ & .728 & $1 / 2$ & 900 & 1256.70 & .513 \\
\hline 2 & 900 & $1,434.68$ & .792 & 2 & 900 & $-1,798.23$ & .707 \\
\hline $1 / 2$ & -100 & -122.63 & .665 & $1 / 2$ & -100 & -140.43 & .507 \\
\hline 2 & -100 & -185.96 & .733 & 2 & -100 & -202.73 & .702 \\
\hline $1 / 2$ & -900 & -999.60 & .811 & $1 / 2$ & -900 & -972.14 & .857 \\
\hline 2 & -900 & $-1,134.70$ & .891 & 2 & -900 & $-1,172.60$ & .876 \\
\hline $1 / 2$ & 70.39 & 100 & .496 & $1 / 2$ & 74.44 & 100 & .554 \\
\hline 2 & 44.94 & 100 & .670 & 2 & 61.27 & 100 & .783 \\
\hline $1 / 2$ & 659.72 & 900 & .537 & $1 / 2$ & 735.40 & 900 & .668 \\
\hline 2 & 493.62 & 900 & .741 & 2 & 653.14 & 900 & .852 \\
\hline $1 / 2$ & -86.23 & -100 & .744 & $1 / 2$ & -62.47 & -100 & .390 \\
\hline 2 & -61.53 & -100 & .784 & 2 & -53.56 & -100 & .732 \\
\hline $1 / 2$ & -757.91 & -900 & .709 & $1 / 2$ & -536.97 & -900 & .356 \\
\hline 2 & -629.56 & -900 & .836 & 2 & -452.68 & -900 & .709 \\
\hline
\end{tabular}

${ }^{\mathrm{a}} N=61$ ( $x_{L}$ matching) and $N=46$ ( $x_{S}$ matching). ${ }^{\mathrm{b}} N=53$ (delay frame) and $N=30$ (speedup frame). ${ }^{\mathrm{c}}$ Delays in years. ${ }^{\mathrm{d}}$ Outcomes in euros. Geometric means. ${ }^{\mathrm{e}}$ Geometric means. 

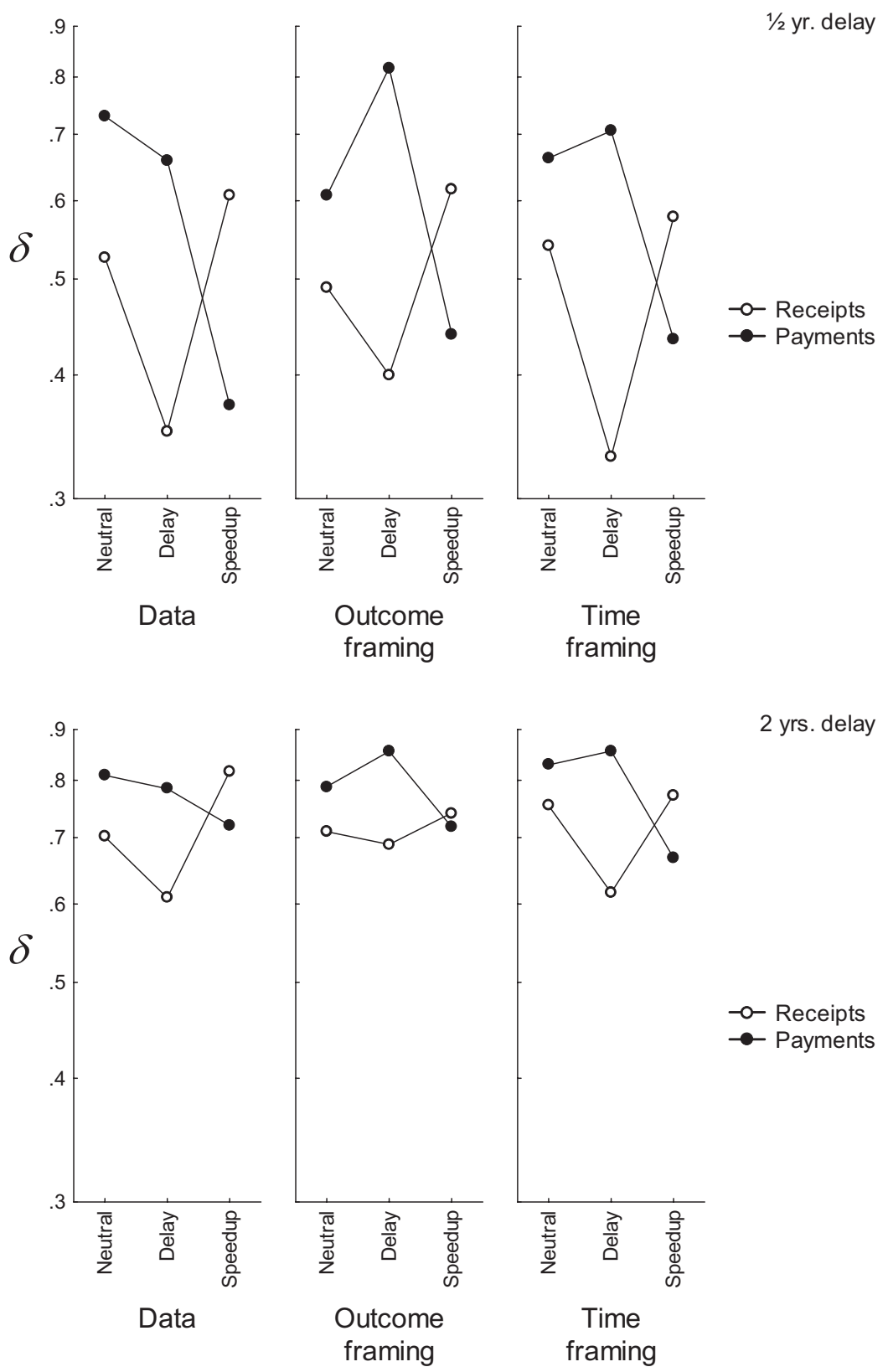

Figure 5. Indifference data from matching. Observed and predicted values of $\delta$ (logarithmic scale). Predictions from the tradeoff models. Neutral frame collapsed across $x_{S}$-matching and $x_{L}$-matching conditions. We observe the delay-speedup asymmetry and the gain-loss asymmetry and, in the comparison between the two panels, the delay effect and the attenuation of the delay-speedup asymmetry for longer delays.

because the overall performance of all seven models, as indexed by the percentage of the variance in the dependent variable unaccounted for, was so much worse. For instance, the badness-of-fit of the time-frame tradeoff model, which came out best in both model contests, was as high as $48 \%$ for our own matching data, but only $19 \%$ for Benzion et al.'s data. So why the noisier data?

We suggest that matching tasks are complicated, and that they were more complicated for our own participants than for Benzion et al.'s (1989) participants. Their participants were students who had completed at least 2-year courses of econom- ics or finance, and were therefore well familiar with the economic theory of discount rates. Most of our own participants also had academic backgrounds, but were probably not as sophisticated in economic and financial matters as Benzion et al.'s students. Economic theory includes two basic discounting effects: Outcomes are discounted by a greater amount over longer intervals than over shorter ones (in Equations 3 and 10, the difference between $x_{L}$ and $x_{S}$ is larger the longer $t_{L}$ ), and larger outcomes are discounted by a greater amount than smaller ones (the difference between $x_{L}$ and $x_{S}$ is larger the 
Table 4

Indifference Data From Matching: Badness-of-Fit Upon Cross-Validating the Discounting Models and the Tradeoff Models on Log( $\delta)$

\begin{tabular}{|c|c|c|c|c|c|}
\hline \multirow[b]{2}{*}{ Model } & \multirow[b]{2}{*}{$k$} & \multicolumn{2}{|c|}{ Experimental trials } & \multicolumn{2}{|c|}{ Rehearsal trial } \\
\hline & & $\mathrm{RMSD}^{\mathrm{a}}$ & $-\log (L)$ & $\mathrm{RMSD}^{\mathrm{b}}$ & $-\log (L)$ \\
\hline Exponential discounting model & 1 & 0.31 & 7.55 & 0.20 & -0.82 \\
\hline Hyperbolic discounting model & 1 & 0.28 & 4.68 & 0.19 & -0.96 \\
\hline Generalized hyperbolic discounting model & 2 & 0.27 & 3.60 & 0.20 & -0.75 \\
\hline Outcome-frame discounting model & 3 & 0.23 & -0.96 & 0.11 & -3.00 \\
\hline Basic tradeoff model & 4 & 0.26 & 2.72 & 0.21 & -0.66 \\
\hline Outcome-frame tradeoff model & 6 & 0.24 & -0.74 & 0.13 & -2.49 \\
\hline Time-frame tradeoff model & 6 & 0.20 & -5.35 & 0.09 & -3.96 \\
\hline
\end{tabular}

${ }^{a}$ The standard deviation of $\log (\delta)$ for the set of 32 data points resulting from a 2 (outcome matched) $\times 2$ (decision frame) $\times 2$ (outcome sign) $\times 2$ (outcome magnitude) $\times 2$ (delay to the larger outcome) design (see Table 3) is 0.30 . $^{\mathrm{b}}$ The standard deviation of $\log (\delta)$ for the set of 4 data points resulting from a 2 (outcome matched) $\times 2$ (decision frame) subdesign is 0.19 .

larger $x_{L}$ ). There is no psychological theory of intertemporal choice that disagrees with this basic logic. However, one third of our participants violated it at least once (see Footnote 4). We excluded these participants from our analyses, but the data from the remaining participants may still have been noisier than the data from Benzion et al.'s students. This is not to devalue model testing in noisy environments, but to explain why the models performed worse on our own data.

It is also very important to emphasize that we fitted our models to per-period discount factors, in contrast with common practice in the experimental literature, which is to fit models to the variable outcome that yields indifference between $S S$ and $L L$, and then report $R^{2}$ (e.g., Green, Myerson, \& Macaux, 2005; Kirby, 1997; Murphy et al., 2001; Rachlin, Raineri, \& Cross, 1991; Scholten \& Read, 2006). The $R^{2}$ values go well into the nineties, with very little discrimination between the models. For instance, in our matching study, the exponential discounting model would have achieved $95.4 \%$ fit, the outcome-frame tradeoff model a $98.2 \%$ fit, and the time-frame tradeoff model a $98.5 \%$ fit: Skyrocketing performance with almost no discrimination between the models. The difference between outcomes, as analyzed in the literature, and per-period discount factors, as analyzed by us, is that the former include basic discounting effects, whereas the latter do not. Because all models, including the exponential discounting model, predict these basic discount- ing effects, the overall performance of the models increases, while the discrimination between the models decreases. To avoid this, one should replace outcomes by per-period discount factors as the dependent variable. Although the exponential discounting model predicts variation in outcomes as far as this variation reflects basic discounting effects, it predicts no variation in per-period discount factors. When estimated and evaluated on $n$ per-period discount factors, it therefore achieves a $0 \%$ fit where the performance of alternative models is usually much better. For instance, in our matching study, the outcomeframe tradeoff model would have achieved a $65.9 \%$ fit, and the time-frame tradeoff model a $75.5 \%$ fit. Overall, per-period discount factors result in a much better discrimination between the models.

\section{Preference Data From Choice}

A problem with the analysis of indifference data from matching is that the delay-speedup asymmetry may be driven by the willingness-to-pay/willingness-to-accept discrepancy: People may understate the compensation they are willing to pay for a desirable change (receiving sooner or paying later, which are time gains in the time-frame tradeoff model) and overstate the compensation they want to receive for an undesirable change (receiving later or paying sooner, which are time losses in the time-frame tradeoff

Table 5

Indifference Data From Matching: Parameter Estimates and Statistical Tests Upon Estimating the Tradeoff Models on Log( $\delta)$

\begin{tabular}{|c|c|c|c|c|c|c|c|}
\hline \multicolumn{4}{|c|}{ Outcome framing } & \multicolumn{4}{|c|}{ Time framing } \\
\hline Parameter & Estimate & $t(26)^{\mathrm{a}}$ & $p$ & Parameter & Estimate & $t(26)^{\mathrm{a}}$ & $p$ \\
\hline \multirow[t]{3}{*}{$\kappa$} & 49.82 & 1.58 & .06 & $\kappa$ & 54.04 & 1.79 & .04 \\
\hline & & & & $\kappa_{l}$ & 1.91 & 3.93 & .00 \\
\hline & & & & $\kappa_{g}$ & 0.87 & -0.86 & .20 \\
\hline$\tau$ & 5.36 & 1.11 & .14 & $\tau^{g}$ & 6.83 & 1.25 & .11 \\
\hline$\gamma$ & 0.02 & 1.95 & .03 & $\gamma$ & 0.02 & 2.50 & .01 \\
\hline$\lambda$ & 1.42 & 2.07 & .02 & $\lambda$ & 1.49 & 2.68 & .01 \\
\hline$\mu_{l}$ & 1.05 & 2.48 & .01 & & & & \\
\hline$\mu_{g}$ & 0.94 & -3.08 & .00 & & & & \\
\hline
\end{tabular}

Note. The models were estimated on 32 data points (see Table 3).

${ }^{a}$ Testing whether $\kappa, \tau$, and $\gamma$ are reliably greater than zero, whether $\kappa_{l}, \lambda$, and $\mu_{l}$ are reliably greater than one, and whether $\kappa_{g}$ and $\mu_{g}$ are reliably smaller than one. 

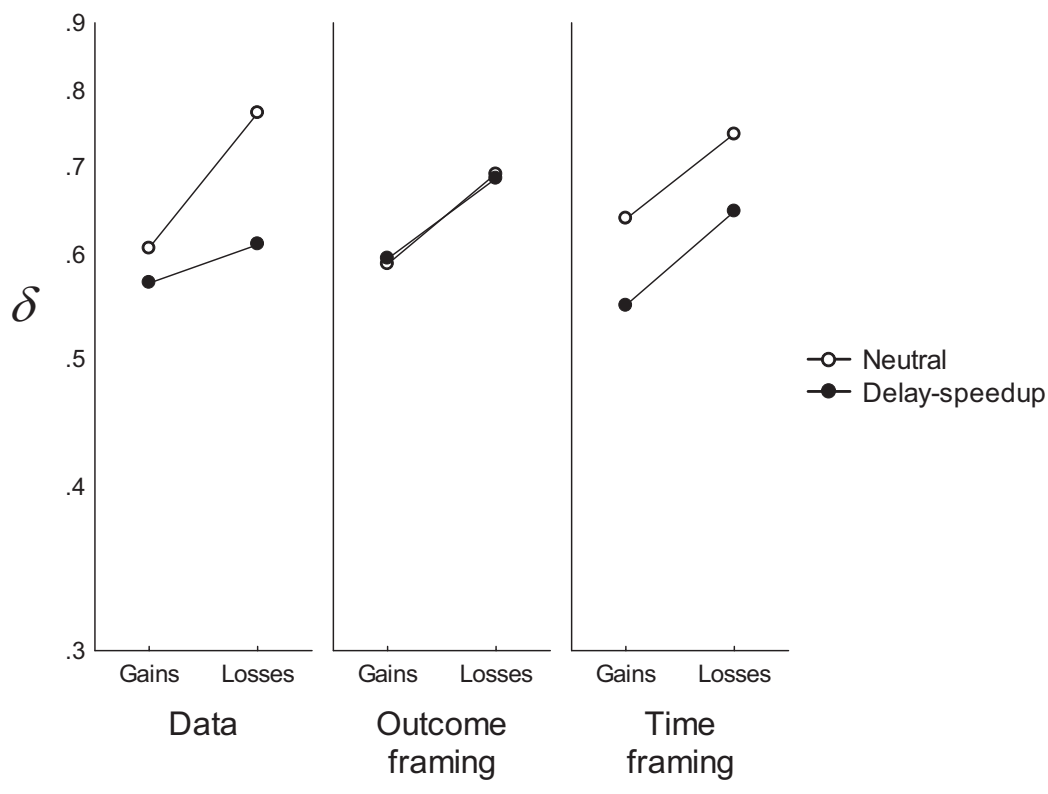

Figure 6. Indifference data from matching. Observed and predicted values of $\delta$ (logarithmic scale). Predictions from the tradeoff models. We observe a less pronounced gain-loss asymmetry and more discounting in the delay-speedup frames than in the neutral frame.

model). According to Loewenstein (1988), straight choice cannot be affected by the willingness-to-pay/willingness-to-accept discrepancy, and so we will conduct an analysis of preference data from choice. In preference data, the delay-speedup asymmetry should show up as inertia, or a bias against changing plans, which is an intertemporal variant of the status-quo bias (Kahneman, Knetsch, \& Thaler, 1991; Ritov \& Baron, 1992; W. Samuelson \& Zeckhauser, 1988).

\section{Method}

Independent and dependent variables. Participants were assigned to one out of 3 (decision frame: neutral, delay, or speedup) conditions. Each participant within these conditions made a choice from each of eight option pairs resulting from a 2 (outcome sign: positive or negative) $\times 2$ (outcome magnitude: small or large) $\times$ 2 (delay to the larger outcome: $1 / 2$ year or 2 years) factorial subdesign. The outcomes were computed as $x_{S}=x_{R}(1+r)^{t_{L} 2}$ and $x_{L}=x_{R}(1+r)^{t_{L} 2}$, where $r=.05$ (with $t_{L}$ expressed in months), $x_{R}=€ 100$ for "small" outcomes, and $x_{R}=€ 500$ (approximately) for "large" outcomes. The outcomes $x_{S}$ and $x_{L}$ were rounded to the nearest $€ 5$. The option pairs are shown in Table 6 .

In the qualitative analysis of experimental effects, the dependent variable is preference for $L L$ (gains) or $S S$ (losses). In the quantitative modeling of the experimental data, the dependent variable is strength of preference, defined as the log-odds of choosing $L L$ (gains) or $S S$ (losses), that is, $\log (\Omega)=\log [P /(1-P)]$, where $P$ is a choice probability. Higher values of $\log (\Omega)$ indicate less discounting. The observed values of $\Omega$ are shown in Table 6 .

Model specification. Model predictions are obtained by taking the logarithm of the ratio between the Right-Hand Side and the Left-Hand Side of the model equations, that is, $\log (\Omega)=\log ($ RHS/ LHS). ${ }^{8}$ For the tradeoff models, in which RHS and LHS represent pairwise advantages, the above specification is equivalent to Restle's (1961) choice model.

We adopt what is known in the literature on risky choice as the representative agent approach (Wu \& Markle, 2008) or singleagent stochastic choice method (Camerer \& Ho, 1994; Wu \& Gonzalez, 1996), which assumes that all participants have the same underlying preferences, but that their choices are stochastically determined by the model equation, that is, the ratio between RHS and LHS. There are two justifications for this approach (see Camerer \& Ho, 1994, for more). First, the participants did not contribute to all data points, only to one third of them, so that the complete models could not have been estimated. Second, intertemporal choice data contain a good deal of noise at the individual level, so that the models would have had great difficulty detecting the signal. These were also our considerations for not analyzing intertemporal matching data at the individual level.

Participants. A total of 298 Portuguese residents, $36 \%$ male and averaging 33 years of age, participated by completing an on-line questionnaire. The recruitment of the participants was the same as in the matching study. Although most e-mails were sent out to persons who had not participated in that study, some overlap between the samples cannot be ruled out. Most participants had an academic degree $(80 \%)$, and most were employed $(61 \%)$ or students $(28 \%)$.

Procedure. The choice tasks were presented as in the examples given in the introduction to this article. Because the delay-speedup frames interchange the order of $S S$ and $L L$, the same was done for the

\footnotetext{
${ }^{8}$ The generic model could also be stated as $\log (\Omega)=\log \left((\mathrm{RHS} / \mathrm{LHS})^{1 / \varepsilon}\right)$, where $\varepsilon$ is a noise parameter (Andersen, Harrison, Lau, \& Rutström, 2010) varying between no noise ( $\varepsilon$ toward zero), in which case choice favors the best option for sure, and only noise ( $\varepsilon$ toward infinity), in which case choice is a coin flip. In the present analyses, $\varepsilon$ consistently converged to its neutral value of 1 .
} 
Table 6

Preference Data From Choice: Delays, Outcomes, and Choice Odds

\begin{tabular}{lcccccc}
\hline & \multicolumn{3}{c}{ Option pairs } & & \multicolumn{3}{c}{$\Omega^{\mathrm{a}}$} \\
\cline { 1 - 2 } \cline { 5 - 6 }$t_{L}{ }^{\mathrm{b}}$ & $x_{S}{ }^{\mathrm{c}}$ & $x_{L}{ }^{\mathrm{c}}$ & & Neutral & Delay & Speedup \\
\hline $1 / 2$ & 85 & 115 & & 0.73 & 0.54 & 1.46 \\
2 & 55 & 180 & & 0.96 & 0.79 & 1.84 \\
$1 / 2$ & 430 & 575 & & 0.93 & 1.00 & 1.46 \\
2 & 280 & 895 & & 3.73 & 2.91 & 2.48 \\
$1 / 2$ & -85 & -115 & & 2.85 & 4.73 & 1.77 \\
2 & -55 & -180 & & 5.50 & 6.82 & 3.00 \\
$1 / 2$ & -430 & -575 & & 3.33 & 3.78 & 1.30 \\
2 & -280 & -895 & & 8.46 & 13.33 & 2.72 \\
\hline
\end{tabular}

${ }^{a} N=104$ (neutral frame), $N=86$ (delay frame), and $N=108$ (speedup frame). ${ }^{\mathrm{b}}$ Delays in years. ${ }^{\mathrm{c}}$ Outcomes in euros.

neutral frame. In the introduction to the questionnaire, participants completed a rehearsal trial. Upon completing the experimental trials, they provided their demographics.

\section{Results}

We stacked the data file, and conducted a factorial probit analysis on preference for $L L$ (gains) or SS (losses). Three factors corresponded to the within-participant subdesign (outcome sign, outcome magnitude, and delay length), and two factors corresponded to the between-participants subdesign: One factor contrasted one presentation order $(S S-L L)$ with the reverse order $(L L-S S)$, the other factor contrasted the neutral frame with the delay-speedup frames. Four main results emerged from this analysis: (1) The delay-speedup and gain-loss asymmetries in the delay-speedup frames and the gain-loss asymmetry in the neutral frame, $\chi^{2}(1)=13.41, p=.00$; (2) the gain-loss asymmetry across frames, $\chi^{2}(1)=130.91, p=.00$; (3) the absolute magnitude effect, $\chi^{2}(1)=17.30, p=.00$; and (4) the delay effect, $\chi^{2}(1)=51.94, p=.00 .^{9}$ The left panels of Figure 7 show the observed values of $\Omega$ along a logarithmic scale, as a measure of the strength of preference for $L L$ (gains) or $S S$ (losses).

Table 7 shows the number of free parameters and the badnessof-fit of the seven models upon $n$-fold cross-validation on the experimental trials. The generalized hyperbolic discounting model could not be estimated, because it went off toward an infinite departure from exponential discounting ( $k$ went toward infinity). Thus, the outcome-frame discounting model incorporates the hyperbolic discount function rather the generalized hyperbolic discount function. Both the outcome-frame discounting model and the hyperbolic discounting model converged on zero discounting $(k$ went toward zero), as did the exponential discounting model ( $r$ went to zero). This is why the badness-of-fit of the hyperbolic discounting model and the exponential discounting model is the same. Going down the list of competing models, badness-of-fit decreases, with the time-frame tradeoff model coming out best.

Table 8 shows the parameter estimates upon estimating the outcome-frame and time-frame tradeoff models on the full set of $n$ data points. Loss aversion $(\lambda)$ is much more pronounced in choice than in matching, reflecting a much larger asymmetry between gains and losses. In the time-frame tradeoff model, hypersensitivity to time lost is more pronounced than hyposensitivity to time gained, $\log \left(\kappa_{l}\right)=0.50>-\log \left(\kappa_{g}\right)=0.33$. In the outcome-frame tradeoff model, however, hypersensitivity to worse than expected states is less pronounced than hyposensitivity to better than expected states, $\log \left(\mu_{l}\right)=0.04<-\log \left(\mu_{g}\right)=0.08$.

The center panels and right panels of Figure 7 show the predictions of the outcome-frame tradeoff model and the time-frame tradeoff model, respectively. For longer delays, we see an attenuation of the delay-speedup asymmetry in gains and an accentuation of the delayspeedup asymmetry in losses, contrary to the findings from matching. The time-frame tradeoff model predicts no moderating effect of delay length, whereas the outcome-frame tradeoff model predicts a pronounced attenuation of the delay-speedup asymmetry in both gains and losses. Omitted from Figure 7 is outcome magnitude. Both tradeoff models closely reproduced the absolute magnitude effect, but not its accentuation for longer delays (see Footnote 9): Neither model predicted a moderating effect of delay length.

Finally, it is again possible to run an additional cross-validation check, because, in the introduction to the questionnaire, the participants performed a rehearsal trial involving medium-large gains ( $€ 165$ and €300) over a medium-length interval (1 year), and this trial can be used for cross-validation. For each model, we used the parameters estimated from the 24 experimental trials to predict $\log (\Omega)$ in the three different rehearsal trials corresponding to the three cells in the 3 (decision frames) between-participants subdesign. This subdesign therefore only includes the delay-speedup asymmetry, and none of the remaining anomalies. The results are shown in Table 7, under the heading "rehearsal trial." The outcome-frame discounting model came out slightly better than the basic tradeoff model in this crossvalidation check. Among the best performing models, the time-frame tradeoff model wins out over the outcome-frame tradeoff model.

\section{General Discussion}

We developed two alternative versions of the tradeoff model (Scholten \& Read, 2010) to address situations in which an outcome is expected at a given time, but then its timing is changed. The outcome framing model proposes that not obtaining a positive outcome when expected is a worse than expected state, to which people are hypersensitive, and that not incurring a negative outcome when expected is a better than expected state, to which people are hyposensitive. The time framing model proposes that delaying a positive outcome or speeding up a negative one is a loss of time, to which people are hypersensitive, and that speeding up a positive outcome or delaying a negative one is a gain of time, to which people are hyposensitive. We compared the two models on their quantitative predictions of indifference data from matching and preference data from choice, and the time framing tradeoff model systematically outperformed the outcome framing model.

From a logical point of view, our findings may come as no surprise: When the situation is a change in the timing of an outcome, then the reaction of people is to the change in timing, not

\footnotetext{
${ }^{9}$ Corollary and secondary results emerged from the probit analysis: (I) An outcome sign by presentation order interaction across decision frames, $\chi^{2}(1)=18.18, p=.00$ (a corollary of Result 1 ); (II) an outcome sign by outcome magnitude interaction, in that the gain-loss asymmetry was more pronounced for small outcomes than for large ones, $\chi^{2}(1)=8.29, p=.00$ (see Loewenstein \& Prelec, 1992); and (III) an accentuation of the absolute magnitude effect for longer delays, $\chi^{2}(1)=8.99, p=.00$.
} 


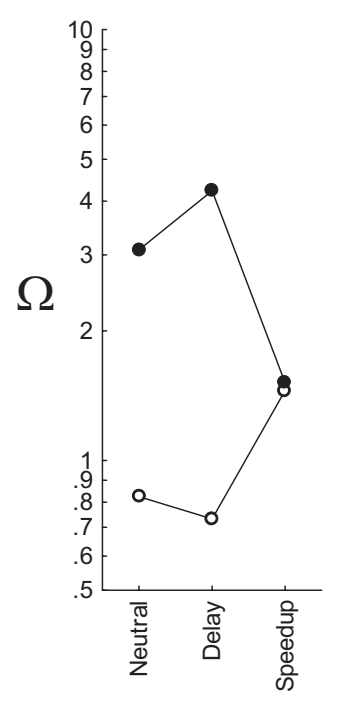

Data

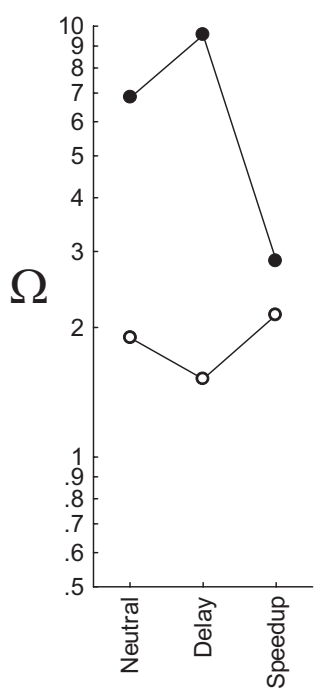

Data
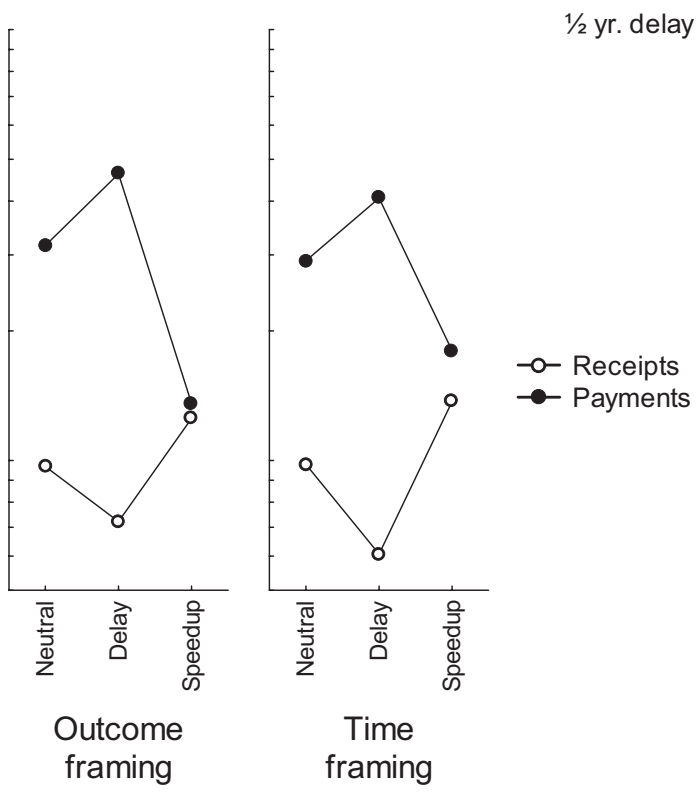

2 yrs. delay
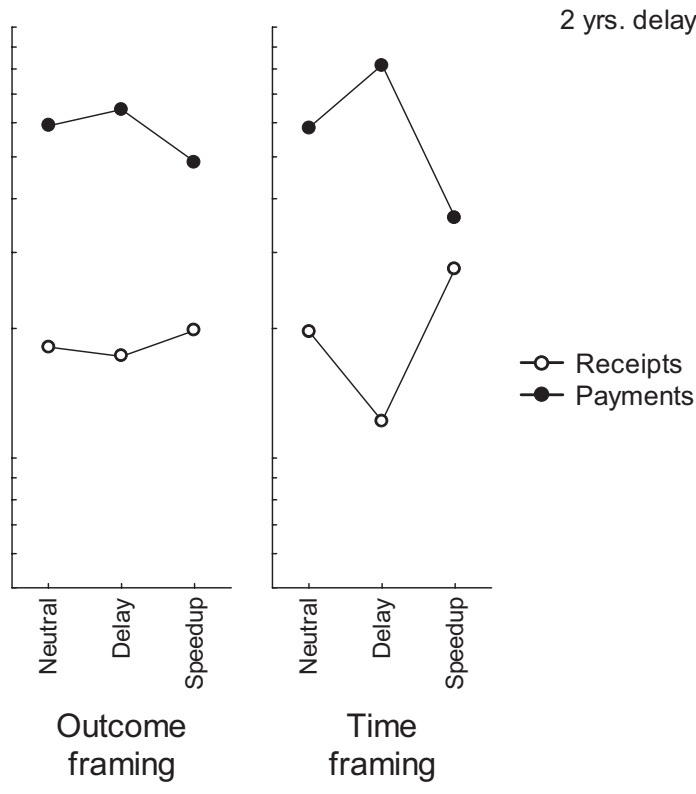

Figure 7. Preference data from choice. Observed and predicted values of $\Omega$ (logarithmic scale). Predictions from the tradeoff models. We observe the delay-speedup asymmetry and the gain-loss asymmetry and, in the comparison between the two panels, the delay effect.

to the outcome whose timing is being changed. However, the time framing model had no precedent in the literature, while the outcome framing model had. The latter generalizes Loewenstein and Prelec's (1992) analysis of the delay-speedup asymmetry, in which not obtaining an expected positive outcome weighs as much as a loss, and not incurring an expected negative outcome weighs as little as a gain. The outcome framing model allows reactions to expected outcomes foregone to be less extreme than that. Even so, its quantitative predictions were systematically less accurate than those of the time framing model.

There is also something strange about the way in which the outcome framing model tried to account for the results: Hypersen- sitivity to worse than expected states was estimated to be less pronounced than hyposensitivity to better than expected states, contrary to ubiquitous evidence showing that "bad is stronger than good" (Baumeister, Bratlavsky, Finkenauer, \& Vohs, 2001). The time framing model, however, acted in accordance with this: Hypersensitivity to time lost was estimated to be more pronounced than hyposensitivity to time gained. The time framing model therefore outperformed the outcome framing model not only in terms of predictive accuracy, but also in terms of interpretability.

So the question arises: Is there any room for outcome framing in the explanation of the delay-speedup asymmetry? Probably not for the kind of framing used by us and others who investigated the 
Table 7

Preference Data From Choice: Badness-of-Fit Upon Cross-Validating the Discounting Models and the Tradeoff Models on Log( $\Omega$ )

\begin{tabular}{|c|c|c|c|c|c|}
\hline \multirow[b]{2}{*}{ Model } & \multirow[b]{2}{*}{$k$} & \multicolumn{2}{|c|}{ Experimental trials } & \multicolumn{2}{|c|}{ Rehearsal trial } \\
\hline & & $\mathrm{RMSD}^{\mathrm{a}}$ & $-\log (L)$ & $\mathrm{RMSD}^{\mathrm{b}}$ & $-\log (L)$ \\
\hline Exponential discounting model & 1 & 0.75 & 27.30 & 0.48 & 2.07 \\
\hline Hyperbolic discounting model & 1 & 0.75 & 27.30 & 0.48 & 2.07 \\
\hline Outcome-frame discounting model & 2 & 0.70 & 25.43 & 0.33 & 0.91 \\
\hline Basic tradeoff model & 4 & 0.54 & 19.34 & 0.38 & 1.33 \\
\hline Outcome-frame tradeoff model ${ }^{\mathrm{c}}$ & 6 & 0.43 & 14.04 & 0.28 & 0.43 \\
\hline Time-frame tradeoff model & 6 & 0.40 & 12.05 & 0.17 & -1.15 \\
\hline
\end{tabular}

${ }^{\text {a }}$ The standard deviation of $\log (\Omega)$ for the set of 24 data points resulting from a 3 (decision frame) $\times 2$ (outcome sign) $\times 2$ (outcome magnitude) $\times 2$ (delay to the larger outcome) design (see Table 6 ) is 0.81 . $^{\mathrm{b}}$ The standard deviation of $\log (\Omega)$ for the set of 3 data points corresponding to the 3 decision frames is 0.38. ${ }^{\mathrm{c}}$ The outcome framing discounting model incorporates Mazur's (1987) hyperbolic discount function rather than the generalized hyperbolic discount function, which could not be estimated.

delay-speedup asymmetry, but other kinds of framing may favor the outcome framing model. For instance, we framed the delay of a receipt as follows: "You are entitled to receive $\$ 100$ today. You can choose to receive $\$ 100$ today as planned, or delay the receipt and receive $\$ 150$ in 1 year." This is a neutral delay frame, in that, a priori, it does not seem to favor either the time framing model or the outcome framing model: It conveys, in the simplest wording possible, the occurrence of a delay. It does, however, leave the foregone gain of $\$ 100$ today implicit, and a biased delay frame can make it explicit, as follows: "You can choose to receive $\$ 100$ today as planned, or delay the receipt and receive $\$ 0$ today but $\$ 150$ in 1 year." By making the foregone gain explicit, the biased delay frame communicates that, when you delay, you will be in a worse than expected state today, which may promote outcome framing, and therefore favor the outcome framing model.

It is not sure that the explicit zero will change the mental process from time framing to outcome framing. Magen, Dweck, and Gross (2008) inserted explicit zeroes into an otherwise neutral frame. Thus, for instance, a choice between "\$100 today" and "\$150 in 1 year" became " $\$ 100$ today and $\$ 0$ in 1 year" and " $\$ 0$ today and $\$ 150$ in 1 year." This increased the popularity of $L L$ over $S S$. An extension of the tradeoff model to sequences accommodates this

Table 8

Preference Data From Choice: Parameter Estimates and Statistical Tests Upon Estimating the Tradeoff Models on $\log (\Omega)$

\begin{tabular}{|c|c|c|c|c|c|c|c|}
\hline \multicolumn{4}{|c|}{ Outcome framing } & \multicolumn{4}{|c|}{ Time framing } \\
\hline Parameter & Estimate & $t(18)^{\mathrm{a}}$ & $p$ & Parameter & Estimate & $t(18)^{\mathrm{a}}$ & $p$ \\
\hline \multirow[t]{3}{*}{$\kappa$} & 56.43 & 1.97 & .03 & $\kappa$ & 55.92 & 1.84 & .04 \\
\hline & & & & $\kappa_{l}$ & 1.62 & 2.17 & .02 \\
\hline & & & & $\kappa_{g}$ & 0.72 & -2.26 & .02 \\
\hline$\tau$ & 3.07 & 1.33 & .10 & $\tau$ & 4.37 & 1.38 & .09 \\
\hline$\gamma$ & 0.01 & 1.92 & .04 & $\gamma$ & 0.01 & 1.96 & .03 \\
\hline$\lambda$ & 3.26 & 4.28 & .00 & $\lambda$ & 2.96 & 4.61 & .00 \\
\hline$\mu_{l}$ & 1.04 & 1.62 & .06 & & & & \\
\hline$\mu_{g}$ & 0.92 & -4.12 & .00 & & & & \\
\hline
\end{tabular}

Note. The models were estimated on 24 data points (see Table 6).

a Testing whether $\kappa, \tau$, and $\gamma$ are reliably greater than zero, whether $\kappa_{l}, \lambda$, and $\mu_{l}$ are reliably greater than one, and whether $\kappa_{g}$ and $\mu_{g}$ are reliably smaller than one. result (Read \& Scholten, 2012), and other known and new anomalies in choices involving sequences. In the extended tradeoff model, a sequence of outcomes is treated as a single dated outcome: It is the sum of the outcomes in the sequence, which is available not at the beginning of the sequence (because the later outcome has yet to occur), and not at the end of the sequence (because the sooner outcome has already occurred), but "somewhere in between." The extended tradeoff model has a mechanism to determine where. Thus, for the sequence " $\$ 100$ today and $\$ 0$ in 1 year," the adjusted delay lies somewhere between today and $1 / 2$ year, and, for the sequence "\$0 today and \$150 in 1 year," the adjusted delay lies somewhere between $1 / 2$ year and 1 year. Thus, the delay of $S S$ has lengthened, the delay of $L L$ has shortened, and we therefore see an increased popularity of $L L$ over $S S$. Plausibly, a person engaged in delay adjustment (which concerns the delay itself) may also become engaged in time framing (which concerns the weight of the delay) when the neutral frame changes into a delay or speedup frame. Thus, we should see the delay-speedup asymmetry, with the explicit zero increasing the willingness to delay a receipt $(L L)$ and decreasing the willingness to speed it up $(S S)$. For payments, it would be the other way around. Clearly, subtleties in framing appear a fertile ground for future research on intertemporal choice.

We benchmarked the performance of the tradeoff models against a number of discounting models, including Mazur's (1987) hyperbolic discount function and Loewenstein and Prelec's (1992) generalized hyperbolic discount function, both very popular devices in research on intertemporal choice. Their performance was very poor, when they are usually portrayed as performing so well. There are several reasons for this.

First, as mentioned earlier, there is the dependent variable: The models are usually fit to the outcomes that yield indifference between the options, not to per-period discount factors at the indifference point. Outcomes include basic discounting effects: They are discounted by a greater amount over longer intervals than over shorter ones, and larger outcomes are discounted by a greater amount than smaller ones. Any model, including the exponential discounting model, predicts this. Inclusion of basic discounting effects in the dependent variable drives up the overall performance of the models, with very little discrimination between them. The per-period discount factors remove basic discounting effects from the dependent variable. The overall performance of the models will 
plunge, with the exponential discounting model being reduced to the null model, because it predicts per-period discount factors to be constant. Hyperbolic discounting models will improve on that by predicting that per-period discount factors will rise with delay length, but their performance will be less impressive than in the prediction of outcomes.

Second, hyperbolic discounting models are usually evaluated on their goodness-of-fit rather than their generalizability. That is, the models are estimated on data, and are then evaluated on their fit to the data on which they were estimated. This also drives up the overall performance of the models, because they will capture not only regularity from the mental process, but also noise. Because the noise changes from one data set to the other, but the regularity does not, the solution is to evaluate the models on their fit to data on which they were not estimated. Generalizability will often be less impressive than goodness-of-fit, with more complex models being penalized insofar as their added complexity captures more noise than regularity.

Third, hyperbolic discounting models are usually evaluated in severely impoverished environments: The magnitude and sign of a delayed outcome is held constant, the delay to the outcome is varied, the immediate outcome that yields indifference between it and the delayed outcome is determined for each delay, and a hyperbolic discounting model is fit to the outcomes that yield indifference. The only anomaly that can happen in this environment is the delay effect, which hyperbolic discounting models accommodate. However, apart from the delay effect and basic discounting effects, they accommodate nothing else. We evaluated hyperbolic discounting models in a more enriched environment, featuring not only the delay effect, but also the absolute magnitude effect, the gain-loss asymmetry, the delay-speedup asymmetry, and its attenuation for longer delays. In such an environment, the hyperbolic discounting models perform poorly, unless they are enriched themselves, such as, in our research, by outcome framing.

The time framing model adds two parameters to a basic tradeoff model that does not accommodate the delay-speedup asymmetry. The addition of these two parameters paid off, because the generalizability of the tradeoff model improved drastically, which means that the added complexity captured more regularity than noise. It thus seems that the time framing model was complex enough to capture regularity and yet simple enough to preclude it would succumb to noise (see Pitt et al., 2002).

The tradeoff model introduces a simple but significant departure from discounting models: Instead of weighing outcome by time, it weighs outcome against time. Different instantiations of a single "master model" account for a wide range of preference patterns in intertemporal choice (Read, Frederick, \& Scholten, 2012; Read \& Scholten, 2012; Scholten \& Read, 2010, 2012). Because outcome is weighted against time, time has value, just as outcome does. Therefore, it is psychologically plausible that, just as people perceive gains and losses along the outcome attribute, they may perceive gains and losses along the time attribute. According to the tradeoff model, they do, and, according to the present development of the model, changes in the timing of an outcome increase the impact of time lost, and decrease the impact of time gained. The analogy between the time and outcome attributes is appealing, but it is imperfect. Outcomes carry value by themselves, time delays do not. Time differences do carry value, just as outcome differences do, but they only acquire value from something good or bad occurring sooner or later. Although time is fundamentally different from outcome, treating time as something that has value as well does seem to improve our understanding of intertemporal choice.

\section{References}

al-Nowaihi, A., \& Dhami, S. (2009). A value function that explains magnitude and sign effects. Economics Letters, 105, 224-229. doi: 10.1016/j.econlet.2009.08.004

Andersen, S., Harrison, G. W., Lau, M. I., \& Rutström, E. E. (2010). Behavioral econometrics for psychologists. Journal of Economic Psychology, 31, 553-576. doi:10.1016/j.joep.2010.03.017

Appelt, K. C., Hardisty, D. J., \& Weber, E. U. (2011). Asymmetric discounting of gains and losses: A query theory account. Journal of Risk and Uncertainty, 43, 107-126. doi:10.1007/s11166-011-9125-1

Baker, F., Johnson, M. W., \& Bickel, W. K. (2003). Delay discounting in current and never-before cigarette smokers: Similarities and differences across commodity, sign, and magnitude. Journal of Abnormal Psychology, 112, 382-392. doi:10.1037/0021-843X.112.3.382

Baumeister, R. F., Bratlavsky, E., Finkenauer, C., \& Vohs, K. D. (2001). Bad is stronger than good. Review of General Psychology, 5, 323-370. doi:10.1037/1089-2680.5.4.323

Benzion, U., Rapoport, A., \& Yagil, J. (1989). Discount rates inferred from decisions: An experimental study. Management Science, 35, 270-284. doi:10.1287/mnsc.35.3.270

Burnham, K. P., \& Anderson, D. R. (2003). Model selection and inference: A practical information-theoretical approach. New York, NY: SpringerVerlag.

Camerer, C. F., \& Ho, T.-H. (1994). Violations of the betweenness axiom and nonlinearity in probability. Journal of Risk and Uncertainty, 8, 167-196. doi:10.1007/BF01065371

Efron, B., \& Tibshirani, R. J. (1993). An introduction to the bootstrap. New York, NY: Chapman \& Hall.

Ganiats, T. G., Carson, R. T., Hamm, R. M., Cantor, S. B., Sumner, W., Spann, S. J., \& Miller, C. (2000). Population-based time preferences for future health outcomes. Medical Decision Making, 20, 263-270. doi: 10.1177/0272989X0002000302

Green, L., Myerson, J., \& Macaux, E. W. (2005). Temporal discounting when the choice is between two delayed rewards. Journal of Experimental Psychology: Learning, Memory, and Cognition, 31, 1121-1133. doi:10.1037/0278-7393.31.5.1121

Kahneman, D., Knetsch, J. L., \& Thaler, R. H. (1991). The endowment effect, loss aversion, and status quo bias. Journal of Economic Perspectives, 5, 193-206. doi:10.1257/jep.5.1.193

Kirby, K. N. (1997). Bidding on the future: Evidence against normative discounting of delayed rewards. Journal of Experimental Psychology: General, 126, 54-70. doi:10.1037/0096-3445.126.1.54

Laibson, D. (1997). Golden eggs and hyperbolic discounting. Quarterly Journal of Economics, 112, 443-478. doi:10.1162/003355397555253

Loewenstein, G. (1988). Frames of mind in intertemporal choice. Management Science, 34, 200-214. doi:10.1287/mnsc.34.2.200

Loewenstein, G., \& Prelec, D. (1992). Anomalies in intertemporal choice: Evidence and an interpretation. Quarterly Journal of Economics, 107, 573-597. doi:10.2307/2118482

Magen, E., Dweck, C. S., \& Gross, J. J. (2008). The hidden-zero effect. Representing a single choice as an extended sequence reduces impulsive choice. Psychological Science, 19, 648-649. doi:10.1111/j.1467-9280 2008.02137.x

Malkoc, S. A., \& Zauberman, G. (2006). Deferring versus expediting consumption: The effect of outcome concreteness on sensitivity to time horizon. Journal of Marketing Research, 43, 618-627. doi:10.1509/ jmkr.43.4.618

Mazur, J. E. (1987). An adjusting procedure for studying delayed rein- 
forcement. In M. L. Commons, J. E. Mazur, L. A. Nevin, \& H. Rachlin (Eds.), Quantitative analyses of behavior V: The effect of delay and of intervening events on reinforcement value (pp. 55-73). Hillsdale, NJ: Erlbaum.

McAlvanah, P. (2010). Subadditivity, patience, and utility: The effects of dividing time intervals. Journal of Economic Behavior \& Organization, 76, 325-337. doi:10.1016/j.jebo.2010.06.009

Murphy, J. G., Vuchinich, R. E., \& Simpson, C. A. (2001). Delayed reward and cost discounting. The Psychological Record, 51, 571-588.

Myung, J. I., Tang, Y., \& Pitt, M. A. (2009). Evaluation and comparison of computational models. Methods in Enzymology, 454, 287-304. doi: 10.1016/S0076-6879(08)03811-1

Pitt, M. A., Myung, J. I., \& Zhang, S. (2002). Toward a method of selecting among computational models of cognition. Psychological Review, 109, 472-491. doi:10.1037/0033-295X.109.3.472

Prelec, D. (2004). Decreasing impatience: A criterion for non-stationary time preference and "hyperbolic" discounting. Scandinavian Journal of Economics, 106, 511-532. doi:10.1111/j.0347-0520.2004.00375.x

Rachlin, H., Raineri, A., \& Cross, D. (1991). Subjective probability and delay. Journal of the Experimental Analysis of Behavior, 55, 233-244. doi:10.1901/jeab.1991.55-233

Read, D., Frederick, S., \& Scholten, M. (2012). DRIFT: An analysis of outcome framing in intertemporal choice. Journal of Experimental Psychology: Learning, Memory, and Cognition. Advance online publication. doi:10.1037/a0029177

Read, D., \& Scholten, M. (2012). Tradeoffs between sequences: Weighing accumulated outcomes against outcome-adjusted delays. Journal of Experimental Psychology: Learning, Memory, and Cognition, 38, 16751688. doi:10.1037/a0028216

Restle, F. (1961). Psychology of judgment and choice: A theoretical essay. New York, NY: Wiley.

Ritov, I., \& Baron, J. (1992). Status-quo and omission bias. Journal of Risk and Uncertainty, 5, 49-61. doi:10.1007/BF00208786

Samuelson, P. A. (1937). A note on measurement of utility. Review of Economic Studies, 4, 155-161. doi:10.2307/2967612

Samuelson, W., \& Zeckhauser, R. (1988). Status quo bias in decision making. Journal of Risk and Uncertainty, 1, 7-59. doi:10.1007/ BF00055564

Scholten, M., \& Read, D. (2006). Discounting by intervals: A generalized model of intertemporal choice. Management Science, 52, 1424-1436. doi:10.1287/mnsc. 1060.0534

Scholten, M., \& Read, D. (2010). The psychology of intertemporal tradeoffs. Psychological Review, 117, 925-944. doi:10.1037/a0019619

Scholten, M., \& Read, D. (2012). Weighing outcome by time or against time? Evaluation rules in intertemporal choice. Manuscript submitted for publication.
Shelley, M. K. (1993). Outcome signs, question frames, and discount rates. Management Science, 39, 806-815. doi:10.1287/mnsc.39.7.806

Spiess, A.-N., \& Neumeyer, N. (2010). An evaluation of $\mathrm{R}^{2}$ as an inadequate measure for nonlinear models in pharmacological and biochemical research: A Monte Carlo approach. BMC Pharmacology, 10, 6. doi: 10.1186/1471-2210-10-6

StatSoft. (2003). Statistica (Version 6) [Computer software]. Tulsa, OK: Author.

Takeuchi, K. (2011). Non-parametric test of time consistency: Present bias and future bias. Games and Economic Behavior, 71, 456-478. doi: 10.1016/j.geb.2010.05.005

Thaler, R. (1980). Toward a positive theory of consumer choice. Journal of Economic Behavior \& Organization, 1, 39-60. doi:10.1016/01672681(80)90051-7

Thaler, R. (1981). Some empirical evidence on dynamic inconsistency. Economics Letters, 8, 201-207. doi:10.1016/0165-1765(81)90067-7

Thaler, R. H., Tversky, A., Kahneman, D., \& Schwartz, A. (1997). The effect of myopia and loss aversion on risk taking: An experimental test. Quarterly Journal of Economics, 112, 647-661. doi:10.1162/ 003355397555226

Tversky, A., \& Kahneman, D. (1991). Loss aversion in riskless choice: A reference-dependent model. Quarterly Journal of Economics, 106, 1039-1061. doi: $10.2307 / 2937956$

Weber, E. U., Johnson, E. J., Milch, K. F., Chang, H., Brodscholl, J. C., \& Goldstein, D. G. (2007). Asymmetric discounting in intertemporal choice: A query-theory account. Psychological Science, 18, 516-523. doi:10.1111/j.1467-9280.2007.01932.x

Wu, G., \& Gonzalez, R. (1996). Curvature of the probability weighting function. Management Science, 42, 1676-1690. doi:10.1287/mnsc.42 .12 .1676

Wu, G., \& Markle, A. B. (2008). An empirical test of gain-loss separability in prospect theory. Management Science, 54, 1322-1335. doi:10.1287/ mnsc. 1070.0846

Xu, L., Liang, Z.-Y., Wang, K., Li, S., \& Jiang, T. (2009). Neural mechanism of intertemporal choice: From discounting future gains to future losses. Brain Research, 1261, 65-74. doi:10.1016/j.brainres.2008 .12 .061

Yates, J. F., \& Watts, R. A. (1975). Preferences for deferred losses Organizational Behavior \& Human Performance, 13, 294-306. doi: 10.1016/0030-5073(75)90051-3

Received May 28, 2012

Revision received October 9, 2012

Accepted October 23, 2012 